\title{
Protecting the stability of the electroweak vacuum from Planck-scale gravitational effects
}

\author{
Vincenzo Branchina, ${ }^{1,2, *}$ Filippo Contino, ${ }^{1,3, \dagger}$ and Apostolos Pilaftsis ${ }^{4, \$}$ \\ ${ }^{1}$ Department of Physics and Astronomy, University of Catania, Via Santa Sofia 64, 95123 Catania, Italy \\ ${ }^{2}$ INFN, Sezione di Catania, Via Santa Sofia 64, 95123 Catania, Italy \\ ${ }^{3}$ Scuola Superiore di Catania, Via Valdisavoia 9, 95123 Catania, Italy \\ ${ }^{4}$ Consortium for Fundamental Physics, School of Physics and Astronomy, \\ University of Manchester, Manchester, M13 9PL, United Kingdom
}

(Received 22 August 2018; published 2 October 2018)

\begin{abstract}
We investigate the stability of the standard model electroweak (EW) vacuum in the presence of Planckscale suppressed operators of the type $\phi^{2 n} / M_{\mathrm{P}}^{2 n-4}$ that involve the Higgs field $\phi$ and could, in principle, be induced by quantum gravity effects. We show how minimal embeddings of the standard model (SM) in supergravity (SUGRA) can stabilize the EW vacuum against such operators up to very high values of the induced supersymmetry breaking scale $M_{\mathcal{S}}$, which may well be above the onset of the so-called SM metastability scale of $10^{11} \mathrm{GeV}$. In particular, we explicitly demonstrate how discrete $R$ symmetries could be invoked to suppress the occurrence of harmful Planck-scale operators of the form $\phi^{2 n} / M_{\mathrm{P}}^{2 n-4}$ to arbitrary higher powers of $n$. We analyze different scenarios of Planck-scale gravitational physics and derive lower limits on the power $n$ that is required in order to protect our EW vacuum from dangerous rapid decay. The significance of our results for theories of low-scale quantum gravity is illustrated.
\end{abstract}

DOI: 10.1103/PhysRevD.98.075001

\section{INTRODUCTION}

The problem of stability of the electroweak (EW) vacuum [1-15] has been central not only in our understanding of the standard model (SM) but also in demystifying the very nature of possible new physics (NP). In particular, recent measurements of the Higgs-boson and the top-quark masses seem to indicate that we live in a metastable Universe, in which the true, absolutely stable vacuum is not the one we now live in, of order $100 \mathrm{GeV}$, but at scales much higher than this, typically larger than $10^{11} \mathrm{GeV}$. Why, then, do we still exist?

Earlier studies that attempted to address this simple but fundamental question were primarily focused on establishing bounds for the Higgs boson mass $M_{H}$. Thus far, three different directions have been followed in the literature: (i) the EW vacuum $v \approx 245 \mathrm{GeV}$ was considered to be absolutely stable, by requiring that the Higgs effective potential $V(\phi)$ does not take values lower than that of the

\footnotetext{
*Branchina@ct.infn.it

†ilippo.contino@ct.infn.it

*Apostolos.Pilaftsis@manchester.ac.uk
}

Published by the American Physical Society under the terms of the Creative Commons Attribution 4.0 International license. Further distribution of this work must maintain attribution to the author(s) and the published article's title, journal citation, and DOI. Funded by SCOAP ${ }^{3}$.
EW minimum; (ii) the EW vacuum has become a metastable state representing a relative minimum of $V(\phi)$, but its lifetime turns out to be larger than the age of the Universe $[2,11,16,17]$; (iii) any possible higher-scale minimum of $V(\phi)$ happens to be degenerate with that of the EW vacuum, obeying some ad hoc principle of multiple criticality [18]. Postulating the latter principle enabled the authors of [18] to obtain predictions for the masses of the Higgs boson $H$ and the top quark $t$, well before their discovery. The so-predicted masses came out to be surprisingly close to their current experimental central values: $M_{t} \simeq 173.34 \mathrm{GeV}$ and $M_{H} \simeq 125.09 \mathrm{GeV}[19,20]$, within the expected theoretical errors [21].

In the context of quantum field theory (QFT), the decay rate of a metastable state (false vacuum) was first calculated by Coleman and Callan, who studied the decay in a flat spacetime background [22,23], and subsequently by Coleman and De Luccia, who included the effect of gravity [24]. The decay is triggered by quantum fluctuations that induce a finite probability for a bubble created at the true vacuum $\phi_{\mathrm{tv}}$ to materialize in a false vacuum $\left(\phi_{\mathrm{fv}}\right)$ sea. Coleman and collaborators considered a scalar theory where the potential $V(\phi)$ has a relative and an absolute minimum at $\phi_{\mathrm{fv}}$ and $\phi_{\mathrm{tv}}$, respectively, with an energy density difference $V\left(\phi_{\mathrm{fv}}\right)-V\left(\phi_{\mathrm{tv}}\right)$ much smaller than the height of the "potential barrier", $V\left(\phi_{\text {top }}\right)-V\left(\phi_{\mathrm{fv}}\right)$, where $V\left(\phi_{\text {top }}\right)$ is the maximum of the potential between the two minima. Given this condition, the true vacuum bubble is 
separated from the false vacuum sea by a "thin wall," and this allows us to treat the problem analytically, within the so-called "thin-wall approximation".

With the discovery of the Higgs boson in 2012, the above question regarding the stability of the $\mathrm{EW}$ vacuum received renewed interest. The goal now is no longer to derive bounds on $M_{H}$ as a function of $M_{t}$, but rather perform precision analyses beyond the leading- and next-to-leadingorder approximation in an attempt to (i) accurately delineate the interfacial region between absolute stability and metastability [25-28], (ii) study the consequences of the EW-vacuum stability on the evolution of the early Universe [29-39], and (iii) test the impact of the latter on the viability of different NP scenarios [25,40-51].

Next-to-next-to-leading-order (NNLO) computations of the SM effective potential $V(\phi)$ show that $t$-quark loops make $V(\phi)$ to turn over for sufficiently large field values of $\phi \gg v$. Taking the central values for $M_{t}$ and $M_{H}$ quoted above at face value, one obtains that a second lower minimum, $\phi_{\mathrm{tv}}$, for the potential $V(\phi)$ gets developed, which is much deeper than the EW one $v \equiv \phi_{\mathrm{fv}}$, for $\phi_{\mathrm{tv}} \gg \phi_{\mathrm{fv}}$. Here, another relevant scale is the so-called metastability or instability scale $\phi_{\text {inst }}$ [1]. The scale $\phi_{\text {inst }}$ is defined as the value, for which $V\left(\phi_{\text {inst }}\right)=V(v)=0$, where the normalization of $V(v)$ to a vanishingly small cosmological constant was considered. Hence, for $\phi>\phi_{\text {inst }}$, the potential $V(\phi)$ becomes negative. For the central values of the Higgs and top masses reported above, one finds that $\phi_{\text {inst }} \sim 10^{11} \mathrm{GeV}$. Moreover, in the SM the thin-wall approximation, i.e., $V\left(\phi_{\mathrm{fv}}\right)-V\left(\phi_{\mathrm{tv}}\right) \ll V\left(\phi_{\mathrm{top}}\right)-V\left(\phi_{\mathrm{fv}}\right)$, may not hold true in general. Consequently, the approximate results of [22,23] and [24] may no longer be applicable. In this case, one is compelled to study this problem numerically.

The decay of the EW vacuum was first studied in a flat spacetime background, by considering the interesting possibility that the SM is valid all the way up to the Planck scale $M_{\mathrm{P}}=1.9 \times 10^{19} \mathrm{GeV}$, and any effects of NP and quantum gravity will show up only at this scale. A crucial assumption of this analysis was that for the calculation of the EW vacuum lifetime $\tau$, the presence of NP operators suppressed by $M_{\mathrm{P}}$ could be neglected [11], and as such, $\tau$ was calculated by considering SM interactions only $[11,12,25,40-44]$. The main reason for this simplification given in [12] was that even if Planck-scale suppressed operators of NP were present, they can still be neglected because the instability scale $\phi_{\text {inst }} \sim 10^{11} \mathrm{GeV}$ happens to be orders of magnitude smaller than $M_{\mathrm{P}}$, thereby tacitly assuming the existence of some kind of a decoupling mechanism.

Restricting ourselves to SM interactions only, the lifetime $\tau$ of the EW vacuum was found to be much larger than the age of our Universe $T_{U} \approx 13.7 \times 10^{9}$ years [25,44], i.e., $\tau \sim 10^{640} T_{U}$ (see [52]). This result is obtained when the effect of standard minimal gravity on the spacetime background metric is ignored. Taking the latter into account, $\tau$ gets "slightly" modified [53-55]: $\tau \sim 10^{660} T_{U}$ (see [52]). Moreover, if a nonminimal interaction $\xi \phi^{2} R$ between the Higgs field $\phi$ and the Ricci scalar $R$ is considered, the lifetime $\tau$ will crucially depend on the value of the nonminimal coupling $\xi$. In particular, in the conformal limit $\xi \rightarrow 1 / 6$, the result of $\tau$ obtained for a flat spacetime background is recovered [55].

In most SM predictions for the lifetime $\tau$ of the EW vacuum, the working hypothesis was that NP operators appearing at scales close to $M_{\mathrm{P}}$ ( $\gg \phi_{\text {inst }}$ ) will decouple and so have no relevant effect on $\tau$. However, this hypothesis turns out to be misleading. It was shown in [45-48] that the presence of such NP operators can drastically modify $\tau$, when considering a flat spacetime background. On the other hand, it is expected on general grounds [24] that the inclusion of gravity will partially counteract the destabilizing effect of any Planckian NP on the EW vacuum. This prompted the authors of [56] to claim that its opposing effect will be so strong, so that the original SM predictions for $\tau$ (i.e., $\tau \sim 10^{660} T_{U}$ ) will be reobtained, thus enabling them to ignore the effect of Planckian NP on $\tau$ altogether. Nevertheless, a recent dedicated analysis does not corroborate such assertions, and the instability of the EW vacuum persists even if gravity is taken into account [52].

It has now become more evident than ever that Planckian NP can strongly affect the stability of the EW vacuum and the presence of possible harmful Planck-scale-suppressed operators of the form $\phi^{2 n} / M_{\mathrm{P}}^{2 n-4}$ can no longer be ignored in the computation. Although we may not be able to exclude a priori such harmful operators, one may still wonder whether a protective symmetry can be invented in order to postpone their appearance to arbitrarily high orders $n$, so as to render their destabilizing effect on the EW vacuum harmless. Our quest is motivated by analogous protective symmetries that were invoked in axion physics to suppress Planck-scale effects and so render the PecceiQuinn mechanism for solving the strong $C P$ problem effective [57-59].

In this paper we will show how supergravity (SUGRA) embeddings of the SM [60] could be sufficient to protect the stability of the EW vacuum up to very large values of the soft supersymmetry (SUSY) breaking scale $M_{\mathcal{S}}$, above the so-called SM metastability scale of $10^{11} \mathrm{GeV}$. Moreover, we will explicitly demonstrate how discrete $R$ symmetries could be used in order to restrict the form of the holomorphic superpotential $\mathcal{W}$, and so suppress the appearance of the harmful Planck-scale operators of the type $\phi^{2 n} / M_{\mathrm{P}}^{2 n-4}$ to arbitrary higher powers of $n$.

The layout of the paper is as follows. After this introductory section, Sec. II provides the necessary theoretical background for our computations of the lifetime $\tau$ of the EW vacuum. In the same section, we use this theoretical background to reaffirm the known predictions for $\tau$ in the SM in the absence of any Planckian NP. In Sec. III, we 
analyze the impact of the harmful operators of the type $\phi^{2 n} / M^{2 n-4}$ on $\tau$, for Planck-scale NP scenarios with $M=$ $M_{\mathrm{P}}$ and $M=M_{\mathrm{P}} / 10$. Section IV discusses minimal embeddings of the SM in SUGRA, which is a wellmotivated theoretical framework that provides good control of Planck-scale physics. We will show how discrete $R$ symmetries could be employed to postpone the appearance of harmful Planck-scale suppressed operators to higher powers of $n$, thus rendering them technically harmless and safe for the stability of the EW vacuum. In Sec. V, we present a numerical analysis of a few representative scenarios with a low and high soft SUSY-breaking scale $M_{\mathcal{S}}$, i.e., for $M_{\mathcal{S}}=10 \mathrm{TeV}$ and $M_{\mathcal{S}}=10^{9} \mathrm{TeV}$. In all our numerical estimates, we consider the effect of gravity on the tunneling rate from the false EW vacuum to the true and absolutely stable trans-Planckian vacuum. Finally, Sec. VI contains our conclusions.

\section{THEORETICAL BACKGROUND}

In this section, we will briefly review the theoretical framework needed for computing the tunneling time $\tau$ from a Minkowski false vacuum $\phi_{\mathrm{fv}}$ to an anti-de Sitter (AdS) true vacuum $\phi_{\mathrm{tv}}$ in the classical, leading-order approximation [22]. With the aid of this framework, we will then be able to reestablish the known results for $\tau$ in the SM, in the absence of any Planckian NP.

\section{A. General framework}

We will now describe the general computational framework for evaluating the lifetime of the EW vacuum $\tau$ by considering first an Euclidean flat spacetime, before turning our attention to an $\mathbb{O}(4)$-symmetric curved background metric.

\section{Euclidean flat spacetime}

Let us first consider the Euclidean flat spacetime action for a real scalar field $\phi$,

$$
S[\phi]=\int d^{4} x\left(\frac{1}{2} \partial_{\mu} \phi \partial_{\mu} \phi+V(\phi)\right),
$$

where $V(\phi)$ denotes the potential with a local minimum (false vacuum) at $\phi=\phi_{\mathrm{fv}}$, and an absolute minimum (true vacuum) at $\phi=\phi_{\mathrm{tv}}$. Taking into account the observed smallness of the cosmological constant, we require that $V\left(\phi_{\mathrm{fv}}\right)=0$, which is an excellent approximation for our purposes.

In order to calculate the lifetime of the false vacuum, we have to first find the so-called "bounce solution" $\phi_{b}(r)$ to the Euler-Lagrange equation of motion for $\phi$ derived from the Euclidean action $S[\phi]$ in (2.1). We note that the bounce solution $\phi_{b}(r)$ is $\mathbb{O}(4)$-symmetric and so depends only on the radial coordinate $r$. Moreover, it has to satisfy certain boundary conditions $[22,23]$. More explicitly, $\phi_{b}(r)$ must be a solution to the Euclidean Euler-Lagrange equation given by

$$
\ddot{\phi}(r)+\frac{3}{r} \dot{\phi}(r)=\frac{d V}{d \phi},
$$

subject to the boundary conditions,

$$
\phi(\infty)=\phi_{\mathrm{fv}}, \quad \dot{\phi}(0)=0 .
$$

Here and in the following, an overdot indicates a derivative with respect to $r$.

Knowing $\phi_{b}(r)$ enables us to evaluate the action $S$ at $\phi_{b}$ as follows:

$$
S\left[\phi_{b}\right]=2 \pi^{2} \int_{0}^{\infty} d r r^{3}\left(\frac{1}{2} \dot{\phi}_{b}^{2}+V\left(\phi_{b}\right)\right) .
$$

In fact, following a reasoning similar to the one used to prove Derrick's theorem, the kinetic term $\frac{1}{2} \dot{\phi}_{b}^{2}$ in (2.4) may effectively be replaced with $-2 V\left(\phi_{b}\right)$, and so a simpler form for the action may be derived, i.e.,

$$
S\left[\phi_{b}\right]=-2 \pi^{2} \int_{0}^{\infty} d r r^{3} V\left(\phi_{b}\right) .
$$

We now have all the ingredients to evaluate the decay rate $\Gamma$ of the false vacuum, which is given by

$$
\Gamma=D \exp \left[-\left(S\left[\phi_{b}\right]-S\left[\phi_{\mathrm{fv}}\right]\right)\right] \equiv D \exp (-B)
$$

where $B \equiv S\left[\phi_{b}\right]-S\left[\phi_{\mathrm{fv}}\right]$ is usually called the "tunneling exponent". The negative of the tunneling exponent, $-B$, determines the leading-order contribution to the decay rate $\Gamma \equiv 1 / \tau$, while $D$ is known as the quantum fluctuation determinant to be discussed below. ${ }^{1}$ Given that $V\left(\phi_{\mathrm{fv}}\right)=0$, the action $S\left[\phi_{\mathrm{fv}}\right]$ vanishes, and the tunneling exponent becomes $B=S\left[\phi_{b}\right]$. In order to determine the lifetime $\tau \equiv$ $1 / \Gamma$ of the false vacuum in the flat spacetime, we first solve numerically (2.2) with boundary conditions (2.3), and then use (2.5) to get the tunneling exponent $B$ of (2.6).

\section{2. $\mathbb{O}(4)$-symmetric curved spacetime}

The next step is to study the impact of gravity on the vacuum decay rate $\Gamma$. To this end, we consider the previous theory in a curved spacetime background, including the Einstein-Hilbert term governed by the Ricci scalar $R$. The Euclidean action now reads

\footnotetext{
${ }^{1}$ The recent renewed interest for the vacuum stability problem prompted a more careful treatment of issues, such as the gauge invariance of the vacuum decay rate and the contribution of zero modes to the quantum fluctuation determinant [61-66].
} 
$S\left[\phi, g_{\mu \nu}\right]=\int d^{4} x \sqrt{g}\left(-\frac{1}{2} M_{\mathrm{Pl}}^{2} R+\frac{1}{2} g^{\mu \nu} \partial_{\mu} \phi \partial_{\nu} \phi+V(\phi)\right)$,

where $g_{\mu \nu}$ is the Euclidean spacetime metric, with $g \equiv$ $\operatorname{det} g_{\mu \nu}$, and $M_{\mathrm{Pl}}$ is the reduced Planck mass. The latter is related to the ordinary Planck mass $M_{\mathrm{P}} \approx 1.9 \times 10^{19} \mathrm{GeV}$ and the Newton's constant $G_{\mathrm{N}}$ as follows:

$$
M_{\mathrm{Pl}}^{2} \equiv \frac{M_{\mathrm{P}}^{2}}{8 \pi}=\left(8 \pi G_{\mathrm{N}}\right)^{-1} .
$$

Requiring now that the curved spacetime metric $g_{\mu \nu}$ be $\mathbb{O}(4)$-symmetric, the (squared) line element may then be expressed as

$$
d s^{2}=d r^{2}+\rho^{2}(r) d \Omega_{3}^{2},
$$

where $d \Omega_{3}^{2}$ is the unit 3-sphere line element and $\rho(r)$ is the volume radius of the 3-sphere at fixed $r$ coordinate [24]. The bounce configuration needed to calculate the false vacuum transition rate will be given now by the field and the metric solutions, $\phi_{b}(r)$ and $\rho_{b}(r)$, to the coupled system of equations:

$$
\ddot{\phi}+3 \frac{\dot{\rho}}{\rho} \dot{\phi}=\frac{d V}{d \phi}, \quad \dot{\rho}^{2}=1+\frac{\kappa \rho^{2}}{3}\left(\frac{1}{2} \dot{\phi}^{2}-V(\phi)\right),
$$

with $\kappa \equiv 8 \pi G_{\mathrm{N}}$. Note that the first equation in (2.10) replaces (2.2), while the second one is the only nontrivial Einstein equation left by $\mathbb{O}(4)$ symmetry. As before, we set $V\left(\phi_{\mathrm{fv}}\right)=0$ at the Minkowski false vacuum $\phi_{\mathrm{fv}}$. As we are interested in the decay of the false vacuum to a true AdS vacuum, the appropriate boundary conditions read

$$
\phi_{b}(\infty)=\phi_{\mathrm{fv}}, \quad \dot{\phi}_{b}(0)=0, \quad \rho_{b}(0)=0 .
$$

We must remark here that besides the nontrivial solution $\left(\phi_{b}(r), \rho_{b}(r)\right)$, the coupled system of equations (2.10) subject to (2.11) also admits a trivial flat-space solution $\phi(r)=\phi_{\mathrm{fv}}$ with a standard Euclidean metric $\rho(r)=r$. Moreover, the first condition in (2.11) implies that as $r \rightarrow \infty$, the bounce $\phi_{b}(r)$ approaches asymptotically the constant false vacuum solution $\phi_{\mathrm{fv}}$, and so $\rho_{b}(r)$ acquires the form of a flat spacetime metric, since one has $\dot{\rho}_{b}(\infty)=$ 1 by virtue of the second equation in (2.10).

With the help of the Einstein equation, $R_{\mu \nu}-\frac{1}{2} g_{\mu \nu} R=$ $\kappa T_{\mu \nu}$, where $T_{\mu \nu}=\partial_{\mu} \phi \partial_{\nu} \phi-g_{\mu \nu}\left(\frac{1}{2} \partial^{\lambda} \phi \partial_{\lambda} \phi+V(\phi)\right)$ is the energy-momentum tensor for the scalar field $\phi$, the Ricci scalar $R$ may then be easily determined by

$$
\frac{1}{\kappa} R=g^{\mu \nu} \partial_{\mu} \phi \partial_{\nu} \phi+4 V(\phi) .
$$

Substituting (2.12) into (2.7) and using the metric (2.9) yields the simpler form for the action

$$
S[\phi, \rho]=-2 \pi^{2} \int_{0}^{\infty} d r \rho^{3} V(\phi) .
$$

Evaluating the action (2.13) for the false vacuum solution $\phi_{\mathrm{fv}}$, we obtain $S\left[\phi_{\mathrm{fv}}, r\right]=0$, leading to the tunneling exponent $B=S_{b} \equiv S\left[\phi_{b}, \rho_{b}\right]$.

Both in the flat and curved spacetime cases, an important parameter is the size $\mathcal{R}$ of the bounce. This is defined as the value of $r$ for which, at $r=\mathcal{R}$, one has

$$
\phi_{b}(\mathcal{R})=\frac{1}{2} \phi_{b}(0) .
$$

By virtue of the bounce size $\mathcal{R}$, the prefactor $D$ in (2.6) may be estimated in the leading-order approximation. In fact, to a good approximation, the lifetime $\tau=\Gamma^{-1}$ of the false EW vacuum may be expressed in terms of $\mathcal{R}$ and the age of Universe $T_{U}$ as follows [67], [11,47]:

$$
\tau \simeq \frac{\mathcal{R}^{4}}{T_{U}^{3}} e^{B}=\frac{\mathcal{R}^{4}}{T_{U}^{4}} e^{S_{b}} T_{U} .
$$

In all our numerical estimates, we will use (2.15) to compute the false vacuum lifetime $\tau$.

\section{B. Stability analysis of the SM}

An application of (2.15) of immediate interest to us is the computation of the lifetime of the EW vacuum of the SM effective potential $V_{\mathrm{SM}}(\phi)$. In this case, the field $\phi$ may be identified with the gauge-invariant radial direction $\phi \equiv \sqrt{2}\left(\Phi^{\dagger} \Phi\right)^{1 / 2}$, where $\Phi$ is the usual SM Higgs doublet. For field values $\phi \gg v$, the renormalization group (RG) improved effective potential may well be approximated as

$$
V_{\mathrm{SM}}(\phi) \approx \frac{1}{4} \lambda_{\mathrm{SM}}(\phi) \phi^{4},
$$

where $\lambda_{\mathrm{SM}}(\phi)$ is the running quartic coupling evaluated at the RG scale $\mu=\phi[2,68]$.

The running quartic coupling $\lambda_{\mathrm{SM}}(\mu)$ has been determined by solving a system of RG equations up to three loop-level accuracy [55], after extending previous studies performed at two loops [25]. As noted in [69], this may lead to certain inconsistencies, when counting the number of loops in the beta functions of the SM interactions, such as those for the Yukawa, gauge and scalar quartic couplings. Since our purpose is to give an order-of-magnitude estimate of the tunneling time $\tau$, we will not use the proposed refinement in [69], as it leads to minimal changes, with no significant impact on the results of our analysis. Instead, we will adopt a more simplified but equally robust approach presented in [70]. In detail, we will approximate the 
TABLE I. Numerical estimates of the lifetime $\tau_{\mathrm{SM}}$ of the SM EW vacuum in the absence of Planckian NP. The values $\phi_{0} \equiv$ $\phi_{b}(0)$ at the center of the bounce and $V_{0} \equiv V_{\mathrm{SM}}\left(\phi_{0}\right)$ are also displayed.

\begin{tabular}{lccc}
\hline \hline Background metric & $\phi_{0} / M_{\mathrm{P}}$ & $V_{0} / M_{\mathrm{P}}^{4}$ & $\tau_{\mathrm{SM}} / T_{U}$ \\
\hline Flat spacetime & 2.306 & $-9.10 \times 10^{-2}$ & $10^{639}$ \\
Curved spacetime & 0.071 & $-8.28 \times 10^{-8}$ & $10^{661}$ \\
\hline \hline
\end{tabular}

RG-improved SM effective potential $V_{\mathrm{SM}}(\phi)$ up to two loops, by fitting the scalar quartic coupling $\lambda_{\mathrm{SM}}(\phi)$ to a three-parameter function:

$$
\lambda_{\mathrm{SM}}(\phi)=\lambda_{*}+\alpha\left(\ln \frac{\phi}{M_{\mathrm{P}}}\right)^{2}+\beta\left(\ln \frac{\phi}{M_{\mathrm{P}}}\right)^{4},
$$

with

$\lambda_{*}=-0.013, \quad \alpha=1.4 \times 10^{-5}, \quad \beta=6.3 \times 10^{-8}$.

Our numerical evaluations will rely on the SM effective potential $V_{\mathrm{SM}}(\phi)$ in (2.16), where $\lambda_{\mathrm{SM}}(\phi)$ is given by (2.17) and (2.18).

Before we carry on studying the impact of NP on the stability of the EW vacuum, we present in Table I the results of our analysis of the tunneling time $\tau$ in the SM: $\tau_{\mathrm{SM}} / T_{U} \sim 10^{639}$ (flat spacetime) and $\tau_{\mathrm{SM}} / T_{U} \sim 10^{661}$ (curved spacetime). These results are in good agreement with those reported in the literature; e.g., see [52]. For completeness, we include in the same table the values of the profile $\phi(r)$ at the center of the bounce, $\phi_{0} \equiv \phi_{b}(0)$, as well as the value $V_{0}$ of the potential (2.16) computed at $\phi_{0}$, i.e., $V_{0} \equiv V_{\mathrm{SM}}\left(\phi_{0}\right)$.

\section{PLANCKIAN NEW PHYSICS EFFECTS}

In all our previous considerations, we have ignored the presence of Planck-scale suppressed operators of the type $\phi^{2 n} / M^{2 n-4}$ in the SM effective potential $V_{\mathrm{SM}}(\phi)$ in (2.16), where $M$ is of order $M_{\mathrm{P}}$. Such operators could in principle be generated by quantum gravity effects and as such, they cannot be excluded a priori from $V_{\mathrm{SM}}(\phi)$. If their contribution to the SM potential happens to be negative, they may have a dramatic destabilizing effect on our EW vacuum, as extensively discussed in [45-48] for $n=3$. For reasons that will become more clear below, we call such operators that contribute with a negative sign to $V_{\mathrm{SM}}(\phi)$ as harmful operators.

Let us consider a set of distinct $\phi^{2 n}$-models that could effectively describe unknown Planckian NP effects. To this end, we extend the SM effective potential as follows:

$$
V_{2 n}(\phi)=V_{\mathrm{SM}}(\phi)+V_{\mathrm{NP}}^{(2 n)}(\phi),
$$

where $n \geq 3$, and

$$
V_{\mathrm{NP}}^{(2 n)}(\phi)=\frac{c_{1}}{2 n} \frac{\phi^{2 n}}{M^{2 n-4}}+\frac{c_{2}}{2(n+1)} \frac{\phi^{2(n+1)}}{M^{2 n-2}} .
$$

Observe that all potentials $V_{2 n}(\phi)$ in (3.1) reduce to $V_{\mathrm{SM}}(\phi)$ for $\phi \ll M$, as its NP part, $V_{\mathrm{NP}}^{(2 n)}(\phi)$, becomes subdominant in this small-field regime. For all the NP effective potentials $V_{\mathrm{NP}}^{(2 n)}(\phi)$, we will assume that $c_{1}$ is negative, but $c_{2}$ is positive, so as to ensure the convexity of the potential at high field values of $\phi \gg M$. Thus, the first term $\phi^{2 n} / M^{2 n-4}$ in (3.2) represents a harmful operator, which we will use from now on to characterize both the $\phi^{2 n}$-model and its effective potential $V_{2 n}(\phi)$.

As an interesting side remark, we note that the NP effective potentials considered in (3.2), as well as the SUGRA effective potentials that we will be studying in Sec. IV, satisfy to a fair degree the much recently discussed Weak Gravity Conjecture (WGC) [71,72]. According to a refined version of the WGC [73,74], consistent effective theories of quantum gravity must satisfy two basic criteria:

$$
\text { (i) } M_{\mathrm{Pl}}\|\nabla V\| \geq c V, \quad \text { (ii) } \Delta \phi \leq d M_{\mathrm{Pl}} \text {. }
$$

Here, $V$ is the effective potential of the candidate theory, $\nabla V$ is its gradient in the field space, $\Delta \phi \sim \phi$ refers to a domain of this field space, and $c$ and $d$ are two positive constants of order 1, whose precise values depend on the details of string compactification. If the two criteria in (3.3) are not met, the candidate theory is said to belong to a kind of swampland, with no possible embedding into a string theory. Rather interestingly, for all the examples considered in the present study, the instanton dynamics, which is crucial here for triggering EW vacuum decay (cf., Sec. II), requires a negative AdS potential, for which $V(\phi) \leq 0$ and $\phi \lesssim M_{\mathrm{P}}=\sqrt{8 \pi} M_{\mathrm{Pl}}$. Hence, for this domain of the $\phi$-space, the first criterion (i) in (3.3) is trivially satisfied, whereas for the second criterion (ii) one only needs to tolerate values of $d$, as large as $\sqrt{8 \pi} \sim 5$. Consequently, for $\phi \gtrsim M_{\mathrm{P}}$, the form of the effective potentials $V_{\mathrm{NP}}^{(2 n)}(\phi)$ needs to be strongly modified according to the WGC criteria in (3.3). However, such modifications to the NP part $V_{\mathrm{NP}}^{(2 n)}(\phi)$ of the SM effective potential at the trans-Planckian regime have no significant effect on the results of our analysis.

In the following, we will analyze numerically the impact of the harmful Planck-scale NP operators $\phi^{2 n} / M^{2 n-4}$ on the tunneling time $\tau$ of the EW vacuum, for all $n \geq 3$. To better assess the relevance of these operators, we will simply set $c_{1}=-2$ and $c_{2}=2$. Moreover, we will investigate two Planck-scale scenarios with: (i) $M=M_{\mathrm{P}}$ and (ii) $M=M_{\mathrm{P}} / 10$, for both a flat and a curved background metric. 

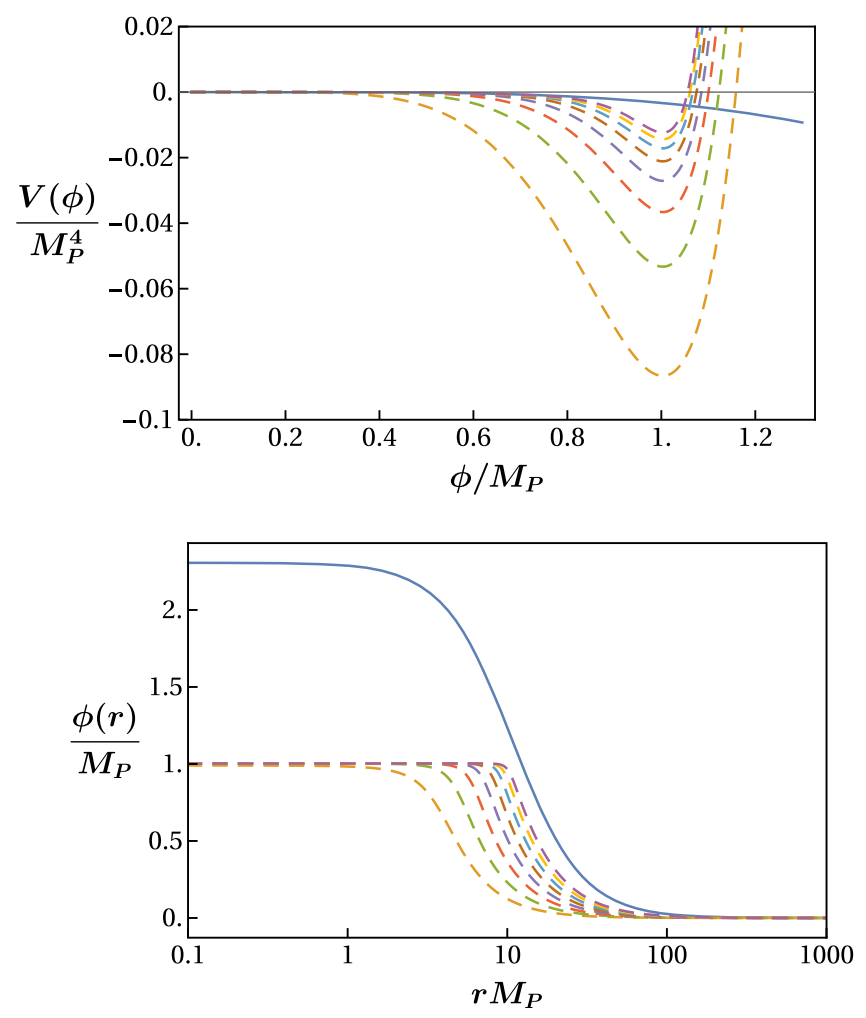

FIG. 1. Upper panel. Scalar potentials $V_{2 n}(\phi)$ [cf. (3.1)], for $3 \leq n \leq 10$, as functions of $\phi$ (dashed lines), with $c_{1}=-c_{2}=$ -2 and $M=M_{\mathrm{P}}$. The solid (blue) line corresponds to the SM potential $V_{\mathrm{SM}}(\phi)$ given in (2.16). Lower panel. Radial profiles of bounce solutions $\phi(r) \equiv \phi_{b}(r)$ (dashed lines) for the same class of Planck-scale scenarios evaluated for a flat spacetime metric. The solid (blue) line refers to the respective SM bounce.

\section{A. Planck-Scale scenarios with $M=M_{\mathrm{P}}$}

We first consider a class of $\phi^{2 n}$-scenarios with scalar potentials $V_{2 n}(\phi)$ given by (3.1), where the Planckian NP scale $M$ is set equal to $M_{\mathrm{P}}$. As can be seen from the upper panel of Fig. 1 and presented by dashed lines in multiple colors, the negative contribution of the harmful Planckscale operators $\phi^{2 n} / M_{\mathrm{P}}^{2 n-4}$ in (3.2) produces a second minimum in their respective effective potentials at $\phi \sim M_{\mathrm{P}}$. For comparison, in the same panel we display with a solid blue line the SM effective potential $V_{\mathrm{SM}}(\phi)$ given in (2.16).

A key quantity that determines the tunneling decay time $\tau$ of the EW vacuum is the actual profile of the bounce solutions $\phi(r) \equiv \phi_{b}(r)$. These are depicted by dashed lines in multiple colors on the lower panel of Fig. 1 for a flat background metric, where the solid line in blue corresponds to the SM bounce. Note that all the bounces $\phi(r)$ reach their highest value close to $r=0$, thereby giving the largest support to the tunneling exponent $B$ in (2.15). When normalizing the effect of the harmful NP operators to the one originating from the SM potential term $\frac{1}{4} \lambda \phi^{4}$, we get the ratio

$$
R_{2 n}=\frac{2 c_{1}}{n \lambda}\left(\frac{\phi(0)}{M_{\mathrm{P}}}\right)^{2 n-4} .
$$

Since $\phi(0) / M_{\mathrm{P}} \sim 1$ for all $n \geq 3$, we expect that as $n$ increases, $R_{2 n}$ will decrease and the predictions for the EW vacuum lifetime $\tau$ will get closer to the SM value $\tau_{\mathrm{SM}}$ presented in Table I. Indeed, this property is observed in Table II for the flat spacetime case. In order to get a lifetime $\tau$ much larger than the age of the Universe $T_{U}$, we need to suppress all potentially harmful operators $\phi^{2 n} / M_{\mathrm{P}}^{2 n-4}$ up to $n=6$, while one gets $\tau \sim \tau_{\mathrm{SM}}$ when $n \geq 50$. In the next section, we will outline a protective mechanism within a SUGRA framework, which can in principle give rise to such a suppression.

Let us now investigate the effect of a curved background metric on the EW vacuum lifetime $\tau$. Unlike in the flat spacetime, an important novel aspect of the curved metric is that for increasing $n$, the bounce solutions $\phi(r)$ and $\rho(r)$ rapidly tend to the corresponding SM bounces, as shown in Fig. 2. As exhibited in Table II, we obtain $\tau \sim \tau_{\mathrm{SM}} \sim$ $10^{661} T_{U}$ (cf. Table I), for all Planck-scale scenarios with $n \geq 4$. This stabilizing effect of gravity on the EW vacuum may also be attributed to the fact that for $n \geq 4$, one finds $\phi(0) \sim 0.07$ which is smaller by more than 1 order of magnitude from the corresponding value in the flat spacetime. As a result, the size of NP contributions as represented by $R_{2 n}$ in (3.4) will decrease more drastically as $n$ grows, for a curved spacetime metric. In the next section, we will explore whether this feature will persist for Planckscale scenarios with a lower quantum gravity scale $M$.

\section{B. Planck-scale scenarios with $M=M_{\mathrm{P}} / \mathbf{1 0}$}

Proceeding as in the previous section, we will analyze a similar class of $\phi^{2 n}$-models, by assuming that the Planckian NP scale $M$ is now 1 order of magnitude smaller, i.e., $M=M_{\mathrm{P}} / 10$. Such a choice may be motivated by the fact that the relevant energy scale of quantum gravity that enters Einstein's equation is the reduced Planck mass $M_{\mathrm{Pl}}$ [cf. (2.8)], rather than the ordinary Planck mass $M_{\mathrm{P}}$.

From the upper panel of Fig. 3, we observe that the minimum of the potentials $V_{2 n}$ is now located to a smaller value at $\phi_{\min } \sim M_{\mathrm{P}} / 10$. The profiles of the bounces $\phi(r)$

TABLE II. Lifetime $\tau$ of the EW vacuum for a class of Planckscale scenarios with harmful operators $\phi^{2 n} / M^{2 n-4}$, evaluated for a flat and a curved background metric. As input values for the NP parameters, we set $c_{1}=-c_{2}=-2$ and $M=M_{\mathrm{P}}$.

\begin{tabular}{lccccr}
\hline \hline$\frac{\phi^{2 n}}{M^{2 n-4}}$ & $\tau / T_{U}$ & $\tau / T_{U}$ & $\frac{\phi^{2 n}}{M^{2 n-4}}$ & $\tau / T_{U}$ & $\tau / T_{U}$ \\
\hline$n$ & (flat) & (curved) & $n$ & (flat) & (curved) \\
\hline 3 & $10^{-208}$ & $10^{-122}$ & 7 & $10^{7}$ & $8.8 \times 10^{661}$ \\
4 & $10^{-166}$ & $3.4 \times 10^{661}$ & 8 & $10^{71}$ & $8.8 \times 10^{661}$ \\
5 & $10^{-114}$ & $8.8 \times 10^{661}$ & 9 & $10^{133}$ & $8.8 \times 10^{661}$ \\
6 & $10^{-55}$ & $8.8 \times 10^{661}$ & 10 & $10^{193}$ & $8.8 \times 10^{661}$ \\
\hline \hline
\end{tabular}



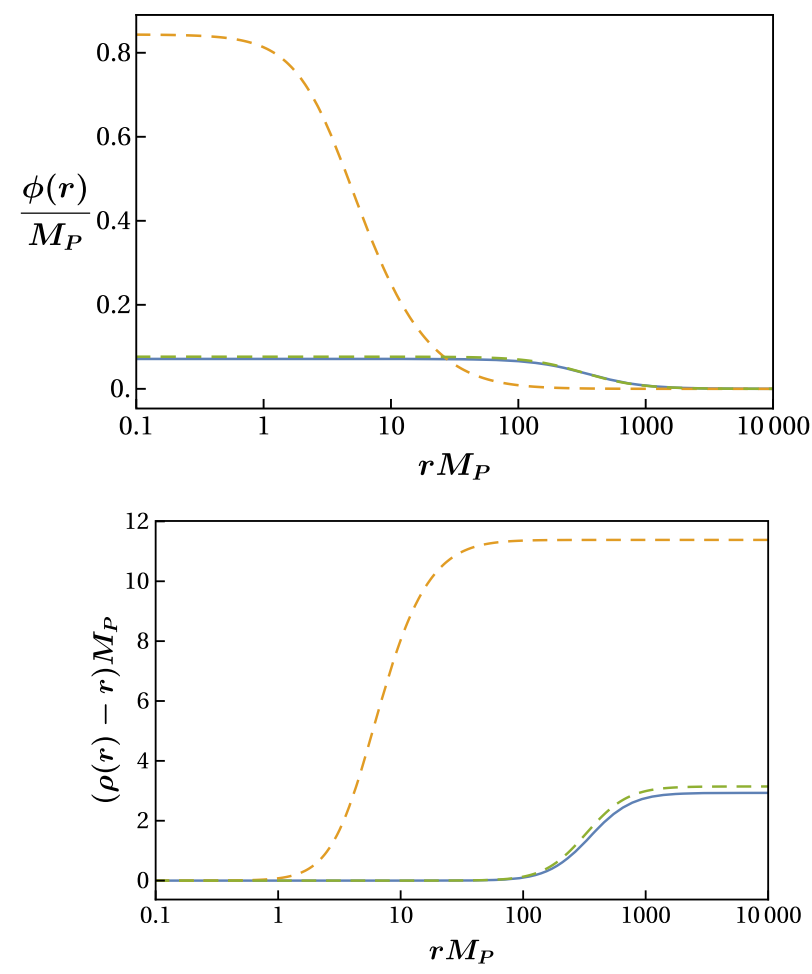

FIG. 2. Upper panel. Radial dependence of the bounce solutions $\phi(r)$ (dashed lines) for the Planck-scale scenarios, with $n=3,4$ and $M=M_{\mathrm{P}}$, evaluated for a curved spacetime metric. Lower panel. Radial profiles for $\rho(r)-r$ (dashed lines) for the same scenarios and background metric. The solid (blue) lines in the two panels show the bounce profiles in the SM.

for a flat spacetime metric are presented by dashed lines in various colors in the lower panel of Fig. 3, while the solid (blue) line stands for the SM bounce. In order to assess the impact of gravity, we give in Table III the lifetime $\tau$ of the EW vacuum, for both a flat and a curved spacetime background. As opposed to the previous scenarios, we now observe that the impact of gravity is less significant, and restoration of the SM prediction for $\tau$ takes place for $n \geq 41$.

Comparing the flat-spacetime results exhibited in Tables III and II, we notice that the predicted values for the tunneling times $\tau$ for each $n$ turn out to be close. As before, we may understand this result by looking at the ratios,

$$
R_{2 n}=\frac{2 c_{1}}{n \lambda}\left(\frac{\phi(0)}{M}\right)^{2 n-4}=\frac{2 c_{1}}{n \lambda}\left(\frac{10 \phi(0)}{M_{\mathrm{P}}}\right)^{2 n-4}
$$

Unlike the previous case $M=M_{\mathrm{P}}$, an extra factor $10^{2 n-4}$ now appears, because we have $M=M_{\mathrm{P}} / 10$. As can be seen from the lower panel of Fig. 3, the maximum of all bounces reached at their origin $(r=0)$ approaches the value: $\phi(0) / M_{\mathrm{P}} \sim 0.1$. Hence, the enhancement factor $10^{2 n-4}$ in (3.5) gets compensated by a corresponding factor
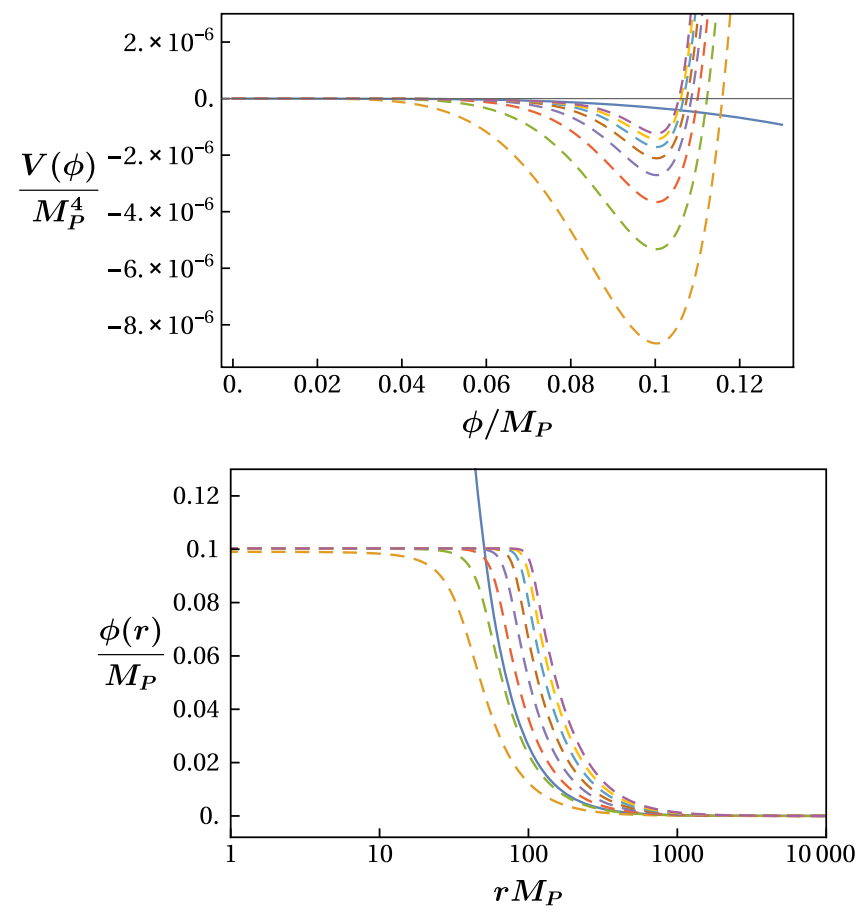

FIG. 3. The same as in Fig. 1, but setting instead $M=M_{\mathrm{P}} / 10$.

$\left(\phi(0) / M_{\mathrm{P}}\right)^{2 n-4} \sim 10^{-(2 n-4)}$. As a consequence of this cancellation, the order-of-magnitude estimates of the tunneling time $\tau$ for the two Planck-scale scenarios, with $M=M_{\mathrm{P}}$ and $M=M_{\mathrm{P}} / 10$, will be comparable.

Let us now turn our attention to the curved spacetime analysis and the numerical estimates of the EW vacuum lifetime $\tau$ given in Table III. As mentioned earlier, the impact of gravity on $\tau$ is minimal in this case. This can be better understood by analyzing the profile for the bounce solutions $\phi(r)$ and $\rho(r)$, for $n \geq 3$. As shown in Fig. 4, the bounces $\phi(r)$ quickly approach the ones found above in Fig. 3 (lower panel) for the flat spacetime metric. Hence, we expect for the EW vacuum lifetime $\tau$ to be less affected by the presence of gravity, becoming independent of the radial coordinate $\rho(r)$.

The above exercise illustrates how the occurrence of harmful operators in Planckian NP theories that happen to

TABLE III. Lifetime $\tau$ of the EW vacuum for a class of Planckscale scenarios that include harmful operators $\phi^{2 n} / M^{2 n-4}$, with $M=M_{\mathrm{P}} / 10$, evaluated for both a flat and a curved background metric.

\begin{tabular}{lccccc}
\hline \hline$\frac{\phi^{2 n}}{M^{2 n-4}}$ & $\tau / T_{U}$ & $\tau / T_{U}$ & $\frac{\phi^{2 n}}{M^{2 n-4}}$ & $\tau / T_{U}$ & $\tau / T_{U}$ \\
\hline$n$ & (flat) & (curved) & $n$ & (flat) & (curved) \\
\hline 3 & $10^{-204}$ & $10^{-203}$ & 7 & $10^{12}$ & $10^{21}$ \\
4 & $10^{-162}$ & $10^{-160}$ & 8 & $10^{76}$ & $10^{87}$ \\
5 & $10^{-110}$ & $10^{-106}$ & 9 & $10^{138}$ & $10^{152}$ \\
6 & $10^{-51}$ & $10^{-44}$ & 10 & $10^{198}$ & $10^{214}$ \\
\hline \hline
\end{tabular}



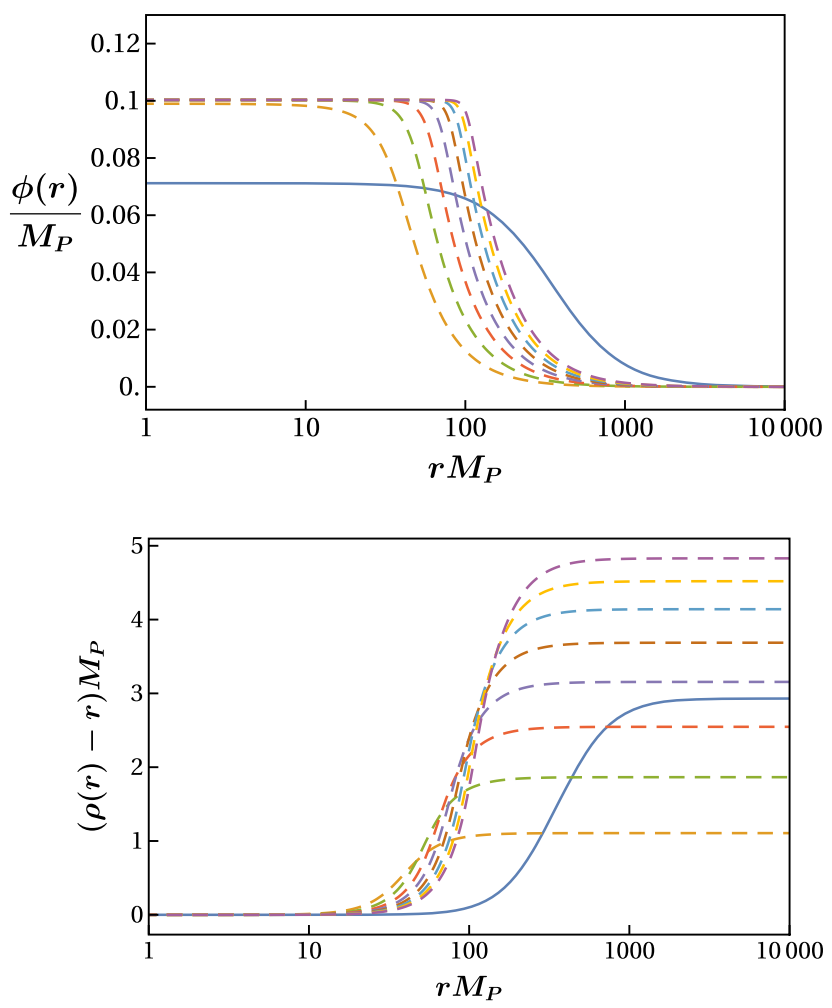

FIG. 4. The same as in Fig. 2, for scenarios with $3 \leq n \leq 10$ and $M=M_{\mathrm{P}} / 10$.

realize a relatively low scale of quantum gravity $M$ face a serious destabilization problem of the EW vacuum. In the next section, we will discuss mechanisms that can naturally suppress the presence of leading harmful operators to sufficiently higher powers of $n$, within a minimal SUGRA framework.

\section{PROTECTIVE MECHANISMS IN SUGRA}

Given our ignorance of a realistic UV-complete theory of quantum gravity, Planck-scale gravitational effects are usually treated within the context of an effective field theory by considering all possible gauge-invariant nonrenormalizable operators suppressed by inverse powers of a high-scale mass $M$, which is typically of the order of the reduced Planck mass $M_{\mathrm{Pl}} \approx 2.4 \times 10^{18} \mathrm{GeV}$. Specifically, gravitational effects on the SM scalar potential $V_{\mathrm{SM}}(\phi)$ along the gauge-invariant field direction $\phi=$ $\sqrt{2}\left(\Phi^{\dagger} \Phi\right)^{1 / 2} \geq 0$, where $\Phi$ is the SM Higgs doublet, may be described by the effective potential

$$
V(\phi)=V_{\mathrm{SM}}(\phi)+\sum_{n=3}^{\infty} \frac{\lambda_{2 n}}{2 n} \frac{\phi^{2 n}}{M^{2 n-4}}
$$

with $V_{\mathrm{SM}}(\phi)=-m^{2} \phi^{2} / 2+\lambda \phi^{4} / 4$. Depending on the sign and size of the coefficient $\lambda_{6}$ for the leading Planck-scale suppressed operator $\phi^{6} / M^{2}$, the lifetime of the EW vacuum can vary by many orders of magnitude [45]. In particular, if $\lambda_{6}$ is negative and $\left|\lambda_{6}\right|$ large, the operator $\phi^{6} / M^{2}$ then becomes harmful and could lead to a dramatic destabilization of the EW vacuum, for both flat and curved spacetime backgrounds [52]. In the following, we will show how SUGRA embeddings of the SM [60] could protect the EW vacuum from rapid decay up to very large values of the soft SUSY-breaking scale $M_{\mathcal{S}}$, above the so-called SM metastability scale of $10^{11} \mathrm{GeV}$.

To start with, let us first consider the minimal supersymmetric extension of the standard model (MSSM), in which only Planck-mass suppressed nonrenormalizable operators involving the Higgs chiral superfields $\hat{H}_{1,2}$ are considered. In other words, for simplicity we ignore nonrenormalizable operators of all other chiral superfields in the effective superpotential $\hat{\mathcal{W}}$. In a SUGRA framework, $\hat{\mathcal{W}}$ will then be given by

$$
\hat{\mathcal{W}}=\hat{\mathcal{W}}_{0}+\mu \hat{H}_{1} \hat{H}_{2}+\sum_{n=2}^{\infty} \frac{\rho_{2 n}}{2 n} \frac{\left(\hat{H}_{1} \hat{H}_{2}\right)^{n}}{M^{2 n-3}}
$$

where

$$
\hat{\mathcal{W}}_{0}=h_{l} \hat{H}_{1} \hat{L} \hat{E}+h_{d} \hat{H}_{1} \hat{Q} \hat{D}+h_{u} \hat{H}_{2} \hat{Q} \hat{U}
$$

is the usual MSSM superpotential without the $\mu$ term, and $\hat{H}_{1}, \hat{H}_{2}$ are the chiral superfields for the two Higgs doublets, $\hat{Q}, \hat{L}$ are the chiral superfields for the quark and lepton left-handed iso-doublets, and $\hat{U}, \hat{D}, \hat{E}$ are their respective right-handed iso-singlet counterparts. Here we follow the conventions of [75]. A comprehensive review of the EW sector of the MSSM is given in [76]. Note that in writing (4.3), we have suppressed all flavor indices from the lepton and quark Yukawa couplings $h_{l}, h_{d}$, and $h_{u}$.

To simplify matters, we consider that all our SUGRA embeddings are based on a minimal Kaehler potential $\hat{\mathcal{K}}$ given by

$$
\hat{\mathcal{K}} \equiv \mathcal{K}\left(\hat{\varphi}_{i}^{*}, \hat{\varphi}_{i}\right)=\hat{H}_{1}^{\dagger} \hat{H}_{1}+\hat{H}_{2}^{\dagger} \hat{H}_{2}+\cdots
$$

where $\hat{\varphi}_{i}$ is a generic chiral superfield and contributions from $\mathrm{SU}(2)_{L}$ and $\mathrm{U}(1)_{Y}$ vector superfields are not shown. At the tree level, the scalar SUGRA potential $V$ may be written as a sum of three terms $-V=V_{F}+V_{D}+V_{\mathrm{br}}-$ since it receives three contributions from (i) $F$ terms $\left(V_{F}\right)$, (ii) $D$ terms $\left(V_{D}\right)$, and (iii) the so-called SUSY-breaking terms $\left(V_{\mathrm{br}}\right)$ induced by spontaneous breakdown of SUGRA that may occur in the so-called hidden sector of the theory [60]. In particular, the $F$ and $D$ terms of the potential $V$ may be calculated from the general expressions: 


$$
\begin{gathered}
V_{F}=e^{\mathcal{K} / M_{\mathrm{Pl}}^{2}}\left[\left(\mathcal{W}_{, i}+\frac{\mathcal{K}_{, i}}{M_{\mathrm{Pl}}^{2}} \mathcal{W}\right) G^{-1, i \bar{j}}\right. \\
\left.\times\left(\mathcal{W}_{, \bar{j}}+\frac{\mathcal{K}_{, \bar{j}}}{M_{\mathrm{Pl}}^{2}} \mathcal{W}^{*}\right)-3 \frac{|\mathcal{W}|^{2}}{M_{\mathrm{Pl}}^{2}}\right], \\
V_{D}=\frac{g^{2}}{2} f_{a b}^{-1} D^{a} D^{b}
\end{gathered}
$$

where $\quad \mathcal{W} \equiv \mathcal{W}\left(\varphi_{i}\right), \quad \mathcal{K} \equiv \mathcal{K}\left(\varphi_{i}^{*}, \varphi_{i}\right), \quad \mathcal{W}_{, i} \equiv \partial \mathcal{W} / \partial \varphi_{i}$ $\mathcal{K}_{, i} \equiv \partial \mathcal{K} / \partial \varphi_{i}, \mathcal{K}_{, i} \equiv \mathcal{K}_{, i}^{*}$ etc, for a generic scalar field $\varphi_{i}$, and $G^{-1, i \bar{j}}$ is the inverse of the Kaehler-manifold metric: $G_{i \bar{j}}=\mathcal{K}_{, i \bar{j}}=\partial^{2} \mathcal{K} /\left(\partial \varphi_{i} \partial \varphi_{j}^{*}\right)$. In addition, $g$ is a generic gauge coupling, e.g., of $\mathrm{SU}(2)_{L}, f_{a b}$ is the gauge kinetic function taken to be minimal, i.e., $f_{a b}=\delta_{a b}$, and $D^{a}=$ $\mathcal{K}_{, \varphi} T^{a} \varphi$ are the so-called $D$ terms, where $T^{a}$ are the generators of the gauge group. Finally, the SUSY-breaking Higgs potential $V_{\mathrm{br}}^{H}$ generated from the effective superpotential in (4.2) is given by

$$
\begin{aligned}
V_{\mathrm{br}}^{H}= & m_{1}^{2}\left|H_{1}\right|^{2}+m_{2}^{2}\left|H_{2}\right|^{2} \\
& +\left(B \mu H_{1} H_{2}+\sum_{n=2}^{\infty} A_{2 n} \frac{\left(H_{1} H_{2}\right)^{n}}{M^{2 n-3}}+\text { H.c. }\right) .
\end{aligned}
$$

For the purpose of this study, we will assume that the $\mu$ term and the soft mass parameters $m_{1,2}^{2}$ and $B \mu$ are of order $M_{\mathcal{S}}$, but all other SUSY-breaking $A$ terms $A_{2 n}$ could be as large as $M$. Although such an unusual assumption does not sizeably destabilize the gauge hierarchy for $M_{\mathcal{S}} \lesssim 10 \mathrm{TeV}$, it can still significantly affect the predictions for the Higgsboson mass spectrum. Here, we will not address the mechanism causing this large hierarchy between the soft parameters and the higher-order $A$ terms, as it strongly depends on the details of the hidden sector in which SUSY gets spontaneously broken [60].

Let us for the moment consider the SUSY limit of the MSSM, by ignoring the induced SUSY-breaking terms $V_{\mathrm{br}}^{H}$ in the scalar potential $V$. Assuming that the $\mu$ term is of order $M_{\mathcal{S}}$ and so negligible when compared to $M_{\mathrm{Pl}}$, the renormalizable part of the MSSM potential, denoted by $V_{0}$, has an $F$ - and $D$-flat direction associated with the gaugeinvariant operator $\hat{H}_{1} \hat{H}_{2}$. In the absence of the $\mu$ term, the scalar field configuration:

$$
H_{1}=\frac{1}{\sqrt{2}}\left(\begin{array}{l}
\phi \\
0
\end{array}\right), \quad H_{2}=\frac{e^{i \xi}}{\sqrt{2}}\left(\begin{array}{l}
0 \\
\phi
\end{array}\right),
$$

with $\xi \in[0,2 \pi)$ and all other scalar fields taken at the origin, gives rise to an exact flat direction for $V_{0}$, i.e., $\partial V_{0} / \partial \phi=0$. Here $\phi$ is a positive scalar field background with canonical kinetic term that parameterizes the $D$-flat direction. The $C P$-odd angle $\xi$ indicates that the flat directions for $H_{1}$ and $H_{2}$ may also differ by an arbitrary relative phase $\xi$. Hence, the parameters $(\phi, \xi)$ describe fully the $D$-flat direction of interest. Now, in the flat-space limit $M_{\mathrm{Pl}} \rightarrow \infty, V_{0 F}$ is positive, implying that $V_{0}=V_{0 F}+$ $V_{0 D} \geq 0$, where the equality sign holds along a flat direction, such as the $\phi$-direction.

The above property of a non-negative potential will generically persist in the minimal SUGRA for the full observable-sector potential $V$, namely upon the inclusion of gauge-invariant nonrenormalizable operators consisting only of MSSM fields. To see this, we first write the MSSM superpotential $\hat{\mathcal{W}}$ as the sum: $\hat{\mathcal{W}}=\sum_{a} \hat{\mathcal{W}}^{a}$, where $\hat{\mathcal{W}}^{a}$ is an arbitrary superpotential term labeled by the index $a$. Then, we notice that the only negative contribution to $V$ can potentially come from both the cross terms and the last term that occur in $V_{F}$ given in (4.5). In particular, up to an overall positive factor $e^{\mathcal{K} / M_{\mathrm{Pl}}^{2}}$, we have

$$
\begin{aligned}
V_{F} & \supset \sum_{a, b}\left[\left(\frac{\mathcal{K}_{, \bar{i}}}{M_{\mathrm{Pl}}^{2}} \mathcal{W}_{, i}^{a} \mathcal{W}^{b}+\text { H.c. }\right)-3 \frac{\mathcal{W}^{a} \mathcal{W}^{b *}}{M_{\mathrm{Pl}}^{2}}\right] \\
& =\sum_{a, b}\left(N_{a}+N_{b}-3\right) \frac{\mathcal{W}^{a} \mathcal{W}^{b}}{M_{\mathrm{Pl}}^{2}} .
\end{aligned}
$$

In arriving at the last equality in (4.9), we used the fact that $\mathcal{K}_{, i} \mathcal{W}_{, i}^{a}=N_{a} \mathcal{W}^{a}$ in minimal SUGRA, where $N_{a}$ is the number of scalar fields $\varphi_{i}$ present in $\hat{\mathcal{W}}^{a}$. Given that $N_{a, b} \geq 2$ for all superpotential terms in the MSSM, the last expression on the rhs of (4.9) will be non-negative, with a possible exception specific field configurations for which $N_{a} \neq N_{b}$. Thus, barring fine-tuning, the $F$-term potential $V_{F}$ of the observable sector in (4.5) will be non-negative. ${ }^{2}$ Since $V_{D} \geq 0$ as well, the complete MSSM scalar potential, including the infinite series of the nonrenormalizable operators, will generically be non-negative.

The above situation changes drastically, if the SUSYbreaking $A$ terms as given in (4.7) are added to the scalar potential $V$. For illustration, let us consider the minimally extended MSSM superpotential

$$
\hat{\mathcal{W}}=\hat{\mathcal{W}}_{0}+\mu \hat{H}_{1} \hat{H}_{2}+\frac{\rho_{4}}{4} \frac{\left(\hat{H}_{1} \hat{H}_{2}\right)^{2}}{M}
$$

which induces the SUSY-breaking potential

\footnotetext{
${ }^{2}$ Instead, hidden-sector chiral superfields $\hat{Z}$ can lead to a negative contribution to $V_{F}$ via SUSY-breaking effects from a Polonyi-type superpotential $\hat{\mathcal{W}}_{\text {hidden }}=m^{2}(\hat{Z}+\beta)$, for which $N_{a, b} \leq 1$. This negative contribution is even desirable, as it can be used to fine-tune the cosmological constant to its observed small value. Similarly, nonminimal Kaehler potential may also lead to negative contributions to $V_{F}$. A more detailed discussion is given in [60].
} 


$$
\begin{aligned}
V_{4, \mathrm{br}}^{H}= & m_{1}^{2}\left|H_{1}\right|^{2}+m_{2}^{2}\left|H_{2}\right|^{2} \\
& +\left(B \mu H_{1} H_{2}+A_{4} \frac{\left(H_{1} H_{2}\right)^{2}}{M}+\text { H.c. }\right),
\end{aligned}
$$

for the Higgs sector. For simplicity, we envisage a scenario for which $m_{1,2}^{2} \ll B \mu$. Moving along the $D$-flat direction as stated in (4.8), and upon ignoring radiative corrections for field values $\phi>M_{\mathcal{S}}$, the leading part of the scalar potential takes on the simple form:

$$
\begin{aligned}
V_{4}(\phi)= & e^{\phi^{2} / M_{\mathrm{Pl}}^{2}}\left[-\frac{m^{2}}{2} \phi^{2}+\frac{\operatorname{Re}\left(e^{2 i \xi} A_{4}\right)}{2 M} \phi^{4}\right. \\
& \left.+\frac{\left|\rho_{4}\right|^{2}}{8} \frac{\phi^{6}}{M^{2}}\left(1+\frac{5}{32} \frac{\phi^{2}}{M_{\mathrm{Pl}}^{2}}+\frac{1}{32} \frac{\phi^{4}}{M_{\mathrm{Pl}}^{4}}\right)\right],
\end{aligned}
$$

where higher-order terms proportional to $|\mu| / M \lesssim$ $M_{\mathcal{S}} / M \ll 1$ were neglected and $m^{2}=\left.\left|e^{i \xi} B \mu-\right| \mu\right|^{2} \mid$ is arranged to be of the required $\mathrm{EW}$ order. Note that even if $A_{4}>0$, the field direction (4.8) with $\xi=\pi / 2$ will make the coefficient $\operatorname{Re}\left(e^{2 i \xi} A_{4}\right)$ entering the potential $V_{4}$ in (4.12) negative. If $A_{4}$ is comparable to $M$, the quartic coupling $\phi^{4}$ can become both sizeable and negative, giving rise to a potential $V_{4}$ that develops a new minimum of order $M /\left|\rho_{4}\right|$, far away from its SM value. On the other hand, the higher powers $\phi^{6}, \phi^{8}$ and $\phi^{10}$ are all proportional to the positive coefficient $\left|\rho_{4}\right|^{2}$, thereby ensuring the convexity of the potential $V_{4}$. Clearly, this exercise shows that SUSY is rather effective in protecting the stability of the EW vacuum from unknown Planck-scale gravitational effects, unless the induced SUSY-breaking coupling $A_{4}$ happens to be of order $M \sim M_{\mathrm{Pl}}$.

In the following, we wish to argue that SUSY may still be effective for protecting the stability of our EW vacuum, even for extreme scenarios with $A_{2 n} \sim M_{\mathrm{Pl}}$ and $M_{\mathcal{S}}$ above the metastability scale of order $10^{11} \mathrm{GeV}$, along the lines of split-SUSY [77,78]. To this end, let us first consider the following discrete symmetry transformations on the chiral superfields:

$$
\left(\hat{H}_{1}, \hat{H}_{2}, \hat{Q}, \hat{L}\right) \rightarrow \omega\left(\hat{H}_{1}, \hat{H}_{2}, \hat{Q}, \hat{L}\right),
$$

whereas the remaining iso-singlet chiral superfields, $\hat{U}, \hat{D}$ and $\hat{E}$, do not transform. Equation (4.13) implies: $\hat{\mathcal{W}} \rightarrow \omega^{2} \hat{\mathcal{W}}$. If $\omega^{2}=1$, the discrete transformations stated in (4.13) give rise to a global $\mathbf{Z}_{2}$ symmetry, which is automatically satisfied by the complete effective superpotential $\mathcal{W}_{\text {eff }}$ in (4.2) and the minimal Kaehler potential $\mathcal{K}$ in (4.4). For $\omega^{2} \neq 1$, however, the superpotential $\mathcal{W}$ is charged and (4.13) represents a nontrivial discrete $R$ symmetry, which is maintained by an appropriate rotation of the Grassmann-valued coordinates of the SUSY space.

We may now exploit this discrete $R$ symmetry in order to suppress lower powers of the nonrenormalizable operators in the effective superpotential $\hat{\mathcal{W}}$ given in (4.2), as well as the respective $A_{2 n}$ terms induced by $\hat{\mathcal{W}}$. Given that $\hat{H}_{1} \hat{H}_{2} \rightarrow \omega^{2} \hat{H}_{1} \hat{H}_{2}$ under the discrete $R$-symmetry transformations in (4.13), we may now require that

$$
\omega^{2 n}=\omega^{2},
$$

for $n>2$. Note that for $n=1,2$, no nontrivial restrictions on the form of $\hat{\mathcal{W}}$ will arise.

Let us therefore turn our attention to the case with $n=3$ in (4.14). This leads to a scenario realizing the discrete $R$ symmetry $\mathbf{Z}_{4}^{R}$, with $\omega^{4}=1$ and $\omega^{2}=-1 \neq 1$. In this case, $\hat{\mathcal{W}}$ takes on the form:

$$
\begin{aligned}
\hat{\mathcal{W}}= & \hat{\mathcal{W}}_{0}+\mu \hat{H}_{1} \hat{H}_{2}+\frac{\rho_{6}}{6} \frac{\left(\hat{H}_{1} \hat{H}_{2}\right)^{3}}{M^{3}} \\
& +\frac{\rho_{10}}{10} \frac{\left(\hat{H}_{1} \hat{H}_{2}\right)^{5}}{M^{7}}+\cdots
\end{aligned}
$$

In such a minimal SUGRA framework with $R$ symmetry, the induced SUSY-breaking potential for the Higgs sector is expected to be of the form [60]:

$$
\begin{aligned}
V_{6, \mathrm{br}}^{H}= & \left(B \mu H_{1} H_{2}+A_{6} \frac{\left(H_{1} H_{2}\right)^{3}}{M^{3}}\right. \\
& \left.+A_{10} \frac{\left(H_{1} H_{2}\right)^{5}}{M^{7}}+\cdots\right)+ \text { H.c. }
\end{aligned}
$$

As before, we assume for simplicity that the soft SUSYbreaking mass parameters $m_{1,2}^{2}$ are small, i.e., $m_{1,2}^{2} \ll B \mu$, so that they can be ignored. Likewise, we assume that only the leading $\rho_{6}$-coupling and the $A_{6}$ term are sizeable and so relevant. In this case, along the $D$-flat direction (4.8), the scalar potential for $\phi>M_{\mathcal{S}}$ will acquire the simple form

$$
\begin{aligned}
V_{6}(\phi)= & e^{\phi^{2} / M_{\mathrm{Pl}}^{2}}\left[-\frac{m^{2}}{2} \phi^{2}+\frac{\operatorname{Re}\left(e^{3 i \xi} A_{6}\right)}{4 M} \frac{\phi^{6}}{M^{2}}\right. \\
& \left.+\frac{\left|\rho_{6}\right|^{2}}{32} \frac{\phi^{10}}{M^{6}}\left(1+\frac{9}{72} \frac{\phi^{2}}{M_{\mathrm{Pl}}^{2}}+\frac{1}{72} \frac{\phi^{4}}{M_{\mathrm{Pl}}^{4}}\right)\right] .
\end{aligned}
$$

In fact, this last result can be generalized to a discrete $R$ symmetry $\mathbf{Z}_{2 n-2}^{R}$, with $\omega^{2(n-1)}=1$ and $n \geq 3$. In this case, the leading form of the scalar potential $V_{2 n}$ for $\phi>M_{\mathcal{S}}$ becomes

$$
\begin{aligned}
V_{2 n}(\phi)= & e^{\phi^{2} / M_{\mathrm{Pl}}^{2}}\left[-\frac{m^{2}}{2} \phi^{2}+\frac{\operatorname{Re}\left(e^{n i \xi} A_{2 n}\right)}{2^{n-1} M} \frac{\phi^{2 n}}{M^{2(n-2)}}\right. \\
& +\frac{\left|\rho_{2 n}\right|^{2}}{2^{2 n-1}} \frac{\phi^{2(2 n-1)}}{M^{2(2 n-3)}}\left(1+\frac{4 n-3}{2(2 n)^{2}} \frac{\phi^{2}}{M_{\mathrm{Pl}}^{2}}\right. \\
& \left.\left.+\frac{1}{2(2 n)^{2}} \frac{\phi^{4}}{M_{\mathrm{Pl}}^{4}}\right)\right] .
\end{aligned}
$$

In the above, we have also neglected all small terms that are proportional to $|\mu| / M$. If $A_{2 n}>0$, the proper harmful 
$D$-flat direction is obtained for $\xi=\pi / n$, leading to the smallest negative coefficient for the $\phi^{2 n}$ operator in (4.18), since $\operatorname{Re}\left(e^{n i \xi} A_{2 n}\right)=-A_{2 n}<0$.

Before concluding this section, a number of important remarks are in order.

(i) It is essential that a discrete $R$ symmetry is employed and not a continuous one, in order to eliminate the harmful Planck-scale operators $\left(H_{1} H_{2}\right)^{n} / M^{2 n-3}$ in (4.7). At first instance, a continuous $\mathrm{U}(1) R$ symmetry would have forbidden all these operators in the scalar potential for $n \geq 2$. However, this would have led to a nonconvex potential along the $D$-flat direction, thereby exacerbating the problem of stability of the electroweak vacuum.

(ii) One may worry that quantum effects may induce further nonrenormalizable operators which in turn may alter the leading form of the potential in (4.18). However, these operators will have higher dimensionality than the leading harmful operator and also expected to be typically suppressed by an extra loop factor $1 /\left(16 \pi^{2}\right) \approx 10^{-2}$ [79]. Moreover, in Dimensional Regularization (DR), corrections to lowerdimensional operators $\phi^{2 n}$ due to seagull graphs resulting from higher dimensional operators, such as $\phi^{2 n+2}$ and higher, are vanishing, if the field direction $\phi$ is taken to be massless. This is indeed a good approximation, given that $m, M_{\mathcal{S}} \ll M$. Finally, possible corrections to SUSY-invariant $F$-term operators in (4.18) can only occur via two (or even powers) of SUSY-breaking operators. As a consequence, such higher-order corrections will be positive definite and so harmless for the stability of the electroweak vacuum.

(iii) It is known that soft SUSY-breaking bilinear and trilinear terms induced by the renormalizable superpotential $\hat{\mathcal{W}}_{0}$ cannot be arbitrary, as they can induce charge and color breaking (CCB) minima [80]. Here we simply assume that these soft SUSY-breaking operators have been chosen so as to avoid the existence of dangerous CCB vacua close to the EW scale. As usually done in the formulation of the MSSM, we impose an extra nonanomalous matter-parity symmetry, the so-called $R$-parity, to get rid of all renormalizable $(B-L)$-violating operators [81], and so allow for a stable dark matter candidate and also ensure the longevity of the proton. Nevertheless, one may still worry that $(B-L)$-conserving nonrenormalizable superpotential operators, such as $\hat{Q} \hat{Q} \hat{Q} \hat{L}$ and $\hat{U} \hat{U} \hat{D} \hat{E}$, might produce harmful SUSY-breaking operators along their $D$-flat direction $[80,82]$. It is easy to see that such operators are not permitted once the discrete $R$ symmetry in (4.13) is imposed, assuming that $\omega^{2} \neq 1$. However, other harmful nonrenormalizable operators of dimension- 4 can survive, such as $\hat{Q} \hat{U} \hat{Q} \hat{D}$ and $\hat{Q} \hat{U} \hat{L} \hat{E}[80,82]$. In the present analysis, we have assumed that their SUSY-breaking $A$ terms are small enough to trigger transitions into a CCB vacuum, along their $D$-flat direction. Alternatively, one may extend the MSSM by adding an extra singlet and choosing other sets of discrete $R$ symmetries along the lines of [83]. Such explorations lie beyond the scope of the present study.

(iv) Likewise, we did not consider explicit stringtheoretic embeddings of the SUGRA scenarios under investigation. In this respect, we did not address the issue of whether all discrete $R$ symmetries $\mathbf{Z}_{2 n-2}^{R}$ can be made nonanomalous with respect to the SM-gauge and gravitational interactions $[81,84-86]$. Instead, we simply note that for the class of the effective superpotentials $\hat{\mathcal{W}}$ considered in (4.2), one can show [87] that all $R$ symmetries have no mixed anomalies with the $\mathrm{SU}(3)_{c}$ gauge group. Apart from $\mathbf{Z}_{4}^{R}$ [87], higher $R$ symmetries might require the addition of exotic colorless matter to avoid mixed anomalies with the $\mathrm{SU}(2)_{L}$ and $\mathrm{U}(1)_{Y}$ groups of the SM. Finally, gravitational instantons may also break anomalously $R$ symmetries. But, there is no consensus [88] whether these are relevant and to what extent they should be included in the path integral. Because of the lack of accurate quantitative estimates of the effect of gravitational instantons, we will assume that any instanton-induced violation of $R$ symmetries, or explicit violations of these through string compactifications, are subdominant and can therefore be ignored for the purpose of this study.

In the next section, we will use the leading form of the SUGRA-derived potential $V_{2 n}(\phi)$ in (4.18), for field values $\phi>M_{\mathcal{S}}$, in order to assess the stability of the EW vacuum against the presence of harmful Planck-scale suppressed operators.

\section{EW VACUUM STABILITY IN SUGRA MODELS}

In this section, we will analyze the stability of the EW vacuum, upon minimally embedding the SM into an effective SUGRA theory that happens to predict the leading form of Planckian NP. We will consider a SUGRA-derived extension of the SM effective potential $V_{2 n}(\phi)$ for Higgs field values $\phi$ above the soft SUSY-breaking scale $M_{\mathcal{S}}$. This means that for $\phi>M_{\mathcal{S}}$, we will adopt the leading form of the SUGRA potential $V_{2 n}(\phi)$ of (4.18). Instead, for $\phi<M_{\mathcal{S}}$, the SM effective potential $V_{\mathrm{SM}}(\phi)$ given in (2.16) will be regarded to be an accurate approximation of the theory, i.e., $V_{2 n}\left(\phi<M_{\mathcal{S}}\right)=V_{\mathrm{SM}}(\phi)$. Given that the reduced Planck mass $M_{\mathrm{Pl}}$ [cf. (2.8)] becomes the relevant mass scale in SUGRA, all mass parameters will be given in $M_{\mathrm{Pl}}$ units. To simplify further our analysis, we identify the scale $M$ in (4.18) with $M_{\mathrm{Pl}}$, i.e., $M=M_{\mathrm{Pl}}$. 
As for the soft SUSY-breaking scale $M_{\mathcal{S}}$, we consider two different scenarios that realize: (i) a very large $M_{\mathcal{S}}=10^{9} \mathrm{TeV}$; (ii) a relatively low $M_{\mathcal{S}}=10 \mathrm{TeV}$. In all scalar potentials $V_{2 n}(\phi)$, we select the flat direction for which the CP-odd phase $\xi$ in (4.8) is given by $\xi=\pi / n$. This gives rise to a harmful operator $\phi^{2 n} / M_{\mathrm{Pl}}^{2 n-4}$ which has the largest negative contribution to $V_{2 n}(\phi)$. Furthermore, for the induced SUSY-breaking trilinears $A_{2 n}$, we assume that they take the following four discrete values:

$A_{2 n}=M_{\mathrm{Pl}}, \quad M_{\mathrm{Pl}} / 5, \quad M_{\mathrm{Pl}} / 10, \quad M_{\mathrm{Pl}} / 50$.

Finally, we set all superpotential couplings $\rho_{2 n}=1$, for simplicity.

\section{A. SUGRA scenarios with $M_{\mathcal{S}}=10^{9} \mathrm{TeV}$}

We will first consider a minimal SUGRA scenario with $M_{\mathcal{S}}=10^{9} \mathrm{TeV}$. The results of our analysis are exhibited in Table IV, for different values of $n$ corresponding to the SUGRA potentials $V_{2 n}(\phi)$ [cf. (4.18)]. In detail, Table IV shows the value of the AdS vacuum energy $V_{\min } \equiv$ $V_{2 n}\left(\phi_{\min }\right)$ at the Planckian AdS vacuum $\phi_{\min }$, the field values, $\phi_{0}^{\text {flat }}$ and $\phi_{0}^{\text {curved }}$, as determined at the center of the bounce (with $\phi_{0} \equiv \phi_{b}(r=0)$ ), as well as the EW vacuum lifetimes $\tau^{\text {flat }}$ and $\tau^{\text {curved }}$ (in $T_{U}$ units) for a flat and a curved spacetime background, respectively. A key theoretical parameter in our analysis is the SUSY-breaking trilinear coupling $A_{2 n}$, which takes four representative values as stated in (5.1).

TABLE IV. Numerical estimates of the AdS vacuum energy $V_{\min }$ at the AdS vacuum $\phi_{\min }$, the field values $\phi_{0} \equiv \phi_{b}(0)$ at the center of the bounce, the $\mathrm{EW}$ vacuum lifetimes $\tau$ (in $T_{U}$ units) for a flat and a curved spacetime background, in SUGRA scenarios with harmful operators $\phi^{2 n} / M^{2 n-4}$ [cf. (4.18)]. The input parameters for such scenarios are: $M_{\mathcal{S}}=10^{9} \mathrm{TeV}, M=M_{\mathrm{Pl}}$, $\rho_{2 n}=1$, while $A_{2 n}$ takes the four discrete values given in (5.1). All energy scales are given in units of the reduced Planck mass $M_{\mathrm{Pl}}$.

\begin{tabular}{lcrccccc}
\hline \hline$n$ & $A_{2 n}$ & $V_{\min }$ & $\phi_{\min }$ & $\phi_{0}^{\text {flat }}$ & $\phi_{0}^{\text {curved }}$ & $\tau^{\text {flat }}$ & $\tau^{\text {curved }}$ \\
\hline 2 & 1 & -4.1791 & 1.4310 & 1.4281 & 1.4253 & $10^{-238}$ & $10^{-238}$ \\
3 & 1 & -5.1768 & 1.4308 & 1.4308 & 1.4308 & $10^{-238}$ & $10^{-237}$ \\
4 & 1 & -5.6986 & 1.4264 & 1.4264 & 1.4264 & $10^{-238}$ & $10^{-236}$ \\
2 & $1 / 5$ & -0.0133 & 0.7161 & 0.0021 & 0.0019 & $10^{-200}$ & $10^{-200}$ \\
3 & $1 / 5$ & -0.0401 & 0.9790 & 0.9787 & 0.9786 & $10^{-146}$ & $10^{-135}$ \\
4 & $1 / 5$ & -0.0669 & 1.0991 & 1.0991 & 1.0991 & $10^{-129}$ & $10^{-104}$ \\
2 & $1 / 10$ & -0.0014 & 0.5122 & 0.0013 & 0.0013 & $10^{-170}$ & $10^{-170}$ \\
3 & $1 / 10$ & -0.0057 & 0.8268 & 0.8262 & 0.8261 & $10^{75}$ & $10^{100}$ \\
4 & $1 / 10$ & -0.0108 & 0.9809 & 0.9809 & 0.9809 & $10^{193}$ & $10^{260}$ \\
2 & $1 / 50$ & $-9.8 \times 10^{-6}$ & 0.2307 & 0.0008 & 0.0008 & $10^{61}$ & $10^{61}$ \\
3 & $1 / 50$ & -0.00007 & 0.5554 & 0.5543 & 0.5543 & $10^{4205}$ & $10^{4354}$ \\
4 & $1 / 50$ & -0.00018 & 0.7519 & 0.7519 & 0.7519 & $10^{8317}$ & $10^{9056}$ \\
\hline \hline
\end{tabular}

From Table IV, we observe that for $A_{2 n}=M_{\mathrm{Pl}}$, no noticeable stabilizing effect on the EW vacuum was found, notwithstanding the presence of gravity and the induced curved background metric. In fact, we have checked that $\tau^{\text {flat }} \sim \tau^{\text {curved }}$, for very high values of $n$ as well. Although this result may seem counterintuitive, it certainly implies that the protective mechanism presented in Sec. IV appears to be ineffective to assure the stability of our EW vacuum in this case.

As $A_{2 n}$ assumes smaller values as shown in Table IV, e.g., $A_{2 n}=M_{\mathrm{Pl}} / 5$, we notice that unlike $n=2$, the lifetime of the EW vacuum, $\tau^{\text {flat }}$ and $\tau^{\text {curved }}$ evaluated separately for a flat and a curved spacetime metric, gets prolonged, as expected. For all the scenarios with $n=2$, the destabilizing effect of the negative $\phi^{4}$ potential term is so strong that even the inclusion of gravity can no longer alter the value of $\tau$. Otherwise, we anticipate on general grounds that the inclusion of gravity will increase the stability of the EW vacuum for all scenarios $n \geq 3$. However, for the scenario with $A_{2 n}=M_{\mathrm{Pl}} / 5$, all low-order harmful operators with $n=2,3,4$ lead to lifetimes $\tau \ll T_{U}$, as can be seen from Table IV. When $A_{2 n}$ becomes even smaller, i.e., $A_{2 n}=$ $M_{\mathrm{Pl}} / 10$ and $A_{2 n}=M_{\mathrm{Pl}} / 50$, a quicker stabilization of the EW vacuum is achieved and the predicted tunneling time $\tau$ becomes much larger than the age of the Universe $T_{U}$, for all scenarios with $n \geq 3$ and $n \geq 2$, respectively. This result is in agreement with the discussion presented in Sec. III since the negative $A_{2 n}$-dependent contribution of the harmful operators to the potentials $V_{2 n}$ becomes less significant for scenarios with lower values $A_{2 n}$.

Finally, it is interesting to observe that as $n$ increases, the AdS vacuum $\phi_{\min }$ and the bounces, $\phi_{0}^{\text {flat }}$ and $\phi_{0}^{\text {curved }}$ at $r=0$, all start to converge towards the same value: $\phi_{\min }=\phi_{0}^{\text {flat }}=\phi_{0}^{\text {curved }}$. In the same context, we have verified that the whole radial profile $\phi^{\text {flat }}(r)$ will start to coincide with that of $\phi^{\text {curved }}(r)$. In fact, the difference between $\tau^{\text {flat }}$ and $\tau^{\text {curved }}$ found in Table IV will result from the two actions of the bounce solutions [cf. (2.5) and (2.13)],

$$
S_{b}^{\mathrm{flat}}=-2 \pi^{2} \int_{0}^{\infty} d r r^{3} V\left(\phi^{\mathrm{flat}}\right)
$$

and

$$
S_{b}^{\text {curved }}=-2 \pi^{2} \int_{0}^{\infty} d r \rho^{3} V\left(\phi^{\text {curved }}\right),
$$

which determine the tunneling exponent $B$ in (2.15). Hence, the deviation of the curvature of the metric, $\rho=$ $\rho(r)$ (curved spacetime), from the respective flat one, $\rho^{\text {flat }}(r)=r$, will control the difference in the predictions for $\tau^{\text {flat }}$ versus $\tau^{\text {curved }}$.

In Fig. 5, we give a concrete example, where we plot the bounces $\phi(r)$ (upper panel) and the curvatures $\rho(r)$ (lower 

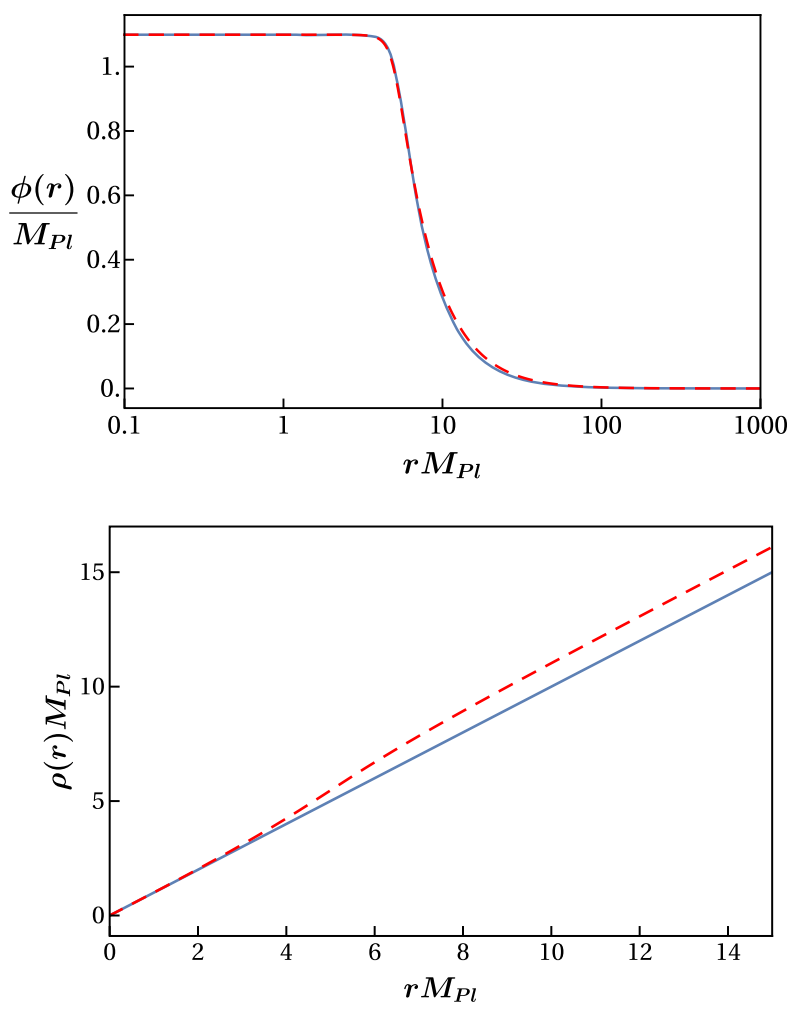

FIG. 5. The bounces $\phi(r)$ (upper panel) and the curvatures $\rho(r)$ (lower panel) for the potential (4.18) with $A_{2 n}=M_{\mathrm{Pl}} / 5$ and $n=4$, for a flat (solid blue) and a curved (dashed red) spacetime.

panel) for the potential (4.18) with $n=4$ and $A_{2 n}=M_{\mathrm{Pl}} / 5$, for a flat (solid blue line) and a curved (dashed red line) background metric. We see that while the two bounce solutions for $\phi(r)$ practically coincide, the corresponding ones for $\rho(r)$ differ from one another, thereby causing the prediction for $\tau^{\text {flat }}$ to significantly deviate from that for $\tau^{\text {curved }}$.

\section{B. SUGRA scenarios with $M_{\mathcal{S}}=10 \mathrm{TeV}$}

We will now study a class of minimal SUGRA scenarios with a soft SUSY-breaking scale $M_{\mathcal{S}}=10 \mathrm{TeV}$. Such scenarios are better motivated, in the sense that they require a much smaller degree of fine tuning for solving the infamous gauge hierarchy problem. Otherwise, all other theoretical parameters take the same values as before. This exercise will allow us to probe the sensitivity of our results to $M_{\mathcal{S}}$. Table V summarizes the findings of our analysis.

As was the case for the SUGRA scenarios with $M_{\mathcal{S}}=10^{9} \mathrm{TeV}$, we find similar features for those with $M_{\mathcal{S}}=10 \mathrm{TeV}$. As before, we obtain that for any fixed value of $A_{2 n}$, the EW vacuum lifetime $\tau$ will increase with $n$. Likewise, $\tau$ will also increase, as $A_{2 n}$ decreases. Similarly, we notice that for $n=2$, the destabilizing effect of the negative $\phi^{4}$ potential term is strong enough to counteract the respective stabilizing effect thanks to gravity. As a consequence, the predictions for $\tau$ turn out not to
TABLE V. The same as in Table IV, but with $M_{\mathcal{S}}=10 \mathrm{TeV}$.

\begin{tabular}{|c|c|c|c|c|c|c|}
\hline$n A_{2 n}$ & $V_{\text {min }}$ & $\phi_{\min }$ & $\phi_{0}^{\text {flat }}$ & $\phi_{0}^{\text {curved }}$ & $\tau^{\text {flat }}$ & $\tau^{\text {curved }}$ \\
\hline 21 & -4.1791 & 1.4310 & 1.4281 & 1.4253 & $10^{-238}$ & $10^{-238}$ \\
\hline 31 & -5.1768 & 1.4308 & 1.4308 & 1.4308 & $10^{-238}$ & $10^{-237}$ \\
\hline 41 & -5.6986 & 1.4264 & 1.4264 & 1.4264 & $10^{-}$ & $10^{-236}$ \\
\hline $21 / 5$ & -0.0133 & 0.7161 & $.24 \times 10^{-}$ & $2.04 \times 10$ & $10^{-184}$ & $10^{-184}$ \\
\hline $31 / 5$ & -0.0401 & 0.9790 & 0.9787 & 0.9786 & $10^{-146}$ & $10^{-135}$ \\
\hline $41 / 5$ & -0.0669 & 1.0991 & 1.0991 & 1.0991 & $10^{-129}$ & $10^{-104}$ \\
\hline $21 / 10$ & -0.0014 & 0.5123 & $49 \times 10^{-}$ & $.47 \times 10^{-}$ & $10^{-154}$ & $10^{-154}$ \\
\hline $31 / 10$ & -0.0057 & 0.8268 & 0.8262 & 0.8261 & $10^{76}$ & $10^{100}$ \\
\hline $41 / 10$ & -0.0108 & 0.9809 & 0.9809 & 0.9809 & $10^{218}$ & $10^{260}$ \\
\hline $21 / 50$ & $.8 \times 10^{-}$ & 0.2 & 10 & $.10 \times 10$ & $10^{76}$ & $10^{76}$ \\
\hline $31 / 50$ & -0.00008 & 0.5554 & 0.5543 & 0.5543 & $10^{4196}$ & $10^{4354}$ \\
\hline $41 / 50$ & -0.00018 & 0.7519 & 0.7519 & 0.7519 & $10^{8006}$ & $10^{9056}$ \\
\hline
\end{tabular}

depend on the choice of the background metric. As before, we observe that for $A_{2 n}=M_{\mathrm{P}} / 50$, the EW vacuum lifetime gets adequately prolonged, becoming much larger than $T_{U}$, already from $n=2$ and on.

If we compare the results presented in Table $\mathrm{V}$ to those in Table IV, we will observe that the tunneling times $\tau^{\text {flat }}$ and $\tau^{\text {curved }}$ are rather comparable, especially when $n \geq 3$. Evidently, this suggests that even if gravity is taken into account, the bounce solutions to (2.2) seem to be insensitive to the matching of the SM effective potential $V_{\mathrm{SM}}(\phi)$ to the SUGRA potential $V_{2 n}(\phi)$ for a wide range of $\phi$ values: $\phi=10-10^{9} \mathrm{TeV}$.

In order to gain further insight into this point, we consider a variant effective scalar potential $\tilde{V}_{2 n}(\phi)$. To be precise, for $\phi<10 \mathrm{TeV}$, we set $\tilde{V}_{2 n}(\phi)=V_{\mathrm{SM}}(\phi)$, and for $\phi>10^{9} \mathrm{TeV}, \tilde{V}_{2 n}(\phi)=V_{2 n}(\phi)$. However, between the field values $\phi=10 \mathrm{TeV}$ and $\phi=10^{9} \mathrm{TeV}$, the new potential $\tilde{V}_{2 n}(\phi)$ is assumed to follow an interpolating straight line. Interestingly enough, the tunneling times $\tau$, as well as the other parameters shown in Table $\mathrm{V}$, come out to be close to the corresponding ones obtained when $V_{\mathrm{SM}}(\phi)$ or $V_{2 n}(\phi)$ are used as an interpolation from 10 to $10^{9} \mathrm{TeV}$. Consequently, the bounce solutions turn out to be insensitive to the shape of the potential in the above range of $\phi$. This observation explains the robustness and the independence of our results for a wide range of soft SUSY-breaking scales: $M_{\mathcal{S}}=10-10^{9} \mathrm{TeV}$.

\section{CONCLUSIONS}

We have studied the stability of the EW vacuum along the radial direction $\phi$ of the Higgs doublet in the presence of Planck-scale suppressed operators of the form $\phi^{2 n} / M^{2 n-4}$, where $M$ is of order the Planck mass $M_{\mathrm{P}}$. Such operators can no longer be excluded in the presence of gravity, as they could in principle be generated by quantum gravity effects. If these operators contribute with a negative 
sign to the SM effective potential, they will in general have a destabilizing effect on the EW vacuum, and therefore we have called them harmful throughout this study.

After briefly describing the theoretical framework for evaluating the lifetime $\tau$ of the EW vacuum in the SM, we have employed it to reestablish the leading-order results for $\tau$ known in the literature (in $T_{U}$ units), i.e., $\tau_{\mathrm{SM}} \sim 10^{639}$ and $\tau_{\mathrm{SM}} \sim 10^{661}$ for a Minkowski and $\mathbb{O}(4)$-symmetric gravitational background metric, respectively. In this theoretical framework, we have then evaluated the lifetime $\tau$ of the EW vacuum for simple scenarios of Planckian NP with convex potentials in the presence of harmful operators. For $n=3$, $M=M_{\mathrm{P}}$ and $c_{2}=c_{1}=-2$ [cf. (3.2)], we recover the recent results quoted in [52], thereby corroborating the importance of the harmful Planck-scale operators on $\tau$, even in the presence of a curved spacetime metric, i.e., $\tau / T_{U} \sim 10^{-122}$. For such scenarios, we have found that longevity of the EW vacuum requires $n \geq 4$, leading to $\tau \gtrsim \tau_{\mathrm{SM}}$. Most remarkably, for theories with relatively lower scale $M$ of quantum gravity, e.g., $M=M_{\mathrm{P}} / 10$, a safely stable $\mathrm{EW}$ vacuum implies that all harmful operators up to $n=6$ need to be either accidentally suppressed or naturally eliminated by the action of some symmetry.

Besides resorting to ad hoc accidental suppressions, in this paper we have explored the possibility whether the harmful Planck-scale operators of the form $\phi^{2 n} / M^{2 n-4}$ could be eliminated naturally to leading order because of the action of some symmetry. In this context, we have shown how minimal embeddings of the SM in SUGRA can stabilize the EW vacuum against these harmful operators up to very high values of the induced SUSY-violating $A$ couplings $A_{2 n}$ and the soft SUSY-breaking scale $M_{\mathcal{S}}$. The scale $M_{\mathcal{S}}$ may even lie above the so-called SM metastability scale of $10^{11} \mathrm{GeV}$. In particular, we have explicitly demonstrated how discrete $R$ symmetries, such as $\mathbf{Z}_{2 n-2}^{R}$, could be invoked to suppress the harmful operators to arbitrary higher powers of $n$. In this minimal SUGRA framework, we have analyzed different scenarios of Planckscale gravitational physics and derived lower limits on the power $n$ that will be needed in order to render the EW vacuum sufficiently long-lived. We have presented numerical estimates for a few representative scenarios realizing a low and high soft SUSY-breaking scale $M_{\mathcal{S}}$, i.e., for $M_{\mathcal{S}}=10 \mathrm{TeV}$ and $M_{\mathcal{S}}=10^{9} \mathrm{TeV}$. In all our numerical estimates, the effect of gravity on the tunneling time $\tau$ from the false EW vacuum to the true Planckian vacuum was carefully considered.

The present study has revealed the severity of a problem for theories with low-scale quantum gravity. In particular, we have illustrated that such theories face serious difficulties in ensuring adequate longevity of our EW vacuum. These theories may have a string-theoretic origin [89] giving rise to realizations with a lower effective Planck mass, including models with large compact dimensions $[90,91]$. It would be interesting to analyze the restrictions that can be derived from the evaluation of $\tau$ on the fundamental parameters of such theories.

\section{ACKNOWLEDGMENTS}

The authors wish to thank the late Maria Krawczyk, acknowledging her warm hospitality at Warsaw University, as well as her strong interest in this study during the early stages of the project. We also thank Elias Kiritsis for useful discussions concerning $\mathrm{R}$ symmetries in string theory. The work of V.B. and F.C. is carried out within the INFN project QFT-HEP and is supported in part by the Polish National Science Centre HARMONIA grant under Contract No. UMO-2015/18/M/ST2/00518. The work of A. P. is supported in part by the Lancaster-ManchesterSheffield Consortium for Fundamental Physics, under STFC Research Grant No. ST/L000520/1.
[1] N. Cabibbo, L. Maiani, G. Parisi, and R. Petronzio, Nucl. Phys. B158, 295 (1979).

[2] R. A. Flores and M. Sher, Phys. Rev. D 27, 1679 (1983).

[3] M. Lindner, Zeitschrift für Physik C Particles and Fields 31, 295 (1986).

[4] M. Sher, Phys. Rep. 179, 273 (1989).

[5] M. Lindner, M. Sher, and H. W. Zaglauer,, Phys. Lett. B 228, 139 (1989).

[6] C. Ford, D. R. T. Jones, P. W. Stephenson, and M. B. Einhorn, Nucl. Phys. B395, 17 (1993).

[7] M. Sher, Phys. Lett. B 317, 159 (1993); 331, 448(A) (1994).

[8] J. A. Casas, J. R. Espinosa, and M. Quiros, Phys. Lett. B 382, 374 (1996).
[9] J. A. Casas, J. R. Espinosa, and M. Quiros, Phys. Lett. B 342, 171 (1995).

[10] G. Altarelli and G. Isidori, Phys. Lett. B 337, 141 (1994).

[11] G. Isidori, G. Ridolfi, and A. Strumia, Nucl. Phys. B609, 387 (2001).

[12] J. R. Espinosa, G. F. Giudice, and A. Riotto, J. Cosmol. Astropart. Phys. 05 (2008) 002.

[13] B.-H. Lee and W. Lee, Classical Quantum Gravity 26, 225002 (2009).

[14] B.-H. Lee, W. Lee, C. Oh, D. Ro, and D.-h. Yeom, J. High Energy Phys. 06 (2013) 003.

[15] B.-H. Lee, W. Lee, D. Ro, and D.-h. Yeom, Phys. Rev. D 91, 124044 (2015). 
[16] M. S. Turner and F. Wilczek, Nature (London) 298, 633 (1982).

[17] P. Hut and M. J. Rees, Nature (London) 302, 508 (1983).

[18] C. D. Froggatt and H. B. Nielsen, Phys. Lett. B 368, 96 (1996).

[19] G. Aad et al. (ATLAS, CMS Collaboration), Phys. Rev. Lett. 114, 191803 (2015).

[20] ATLAS, CDF, CMS, and D0 Collaboration, arXiv:1403 .4427.

[21] A. V. Bednyakov, B. A. Kniehl, A. F. Pikelner, and O. L. Veretin, Phys. Rev. Lett. 115, 201802 (2015).

[22] S. R. Coleman, Phys. Rev. D 15, 2929 (1977); 16, 1248(E) (1977).

[23] C. G. Callan, Jr. and S. R. Coleman, Phys. Rev. D 16, 1762 (1977).

[24] S. R. Coleman and F. De Luccia, Phys. Rev. D 21, 3305 (1980).

[25] G. Degrassi, S. Di Vita, J. Elias-Miro, J. R. Espinosa, G. F. Giudice, G. Isidori, and A. Strumia, J. High Energy Phys. 08 (2012) 098.

[26] B. Garbrecht and P. Millington, Phys. Rev. D 91, 105021 (2015).

[27] B. Grinstein and C. W. Murphy, J. High Energy Phys. 12 (2015) 1.

[28] B. Garbrecht and P. Millington, J. Phys. Conf. Ser. 873, 012041 (2017).

[29] M. Herranen, T. Markkanen, S. Nurmi, and A. Rajantie, Phys. Rev. Lett. 113, 211102 (2014).

[30] N. Khan and S. Rakshit, Phys. Rev. D 90, 113008 (2014).

[31] M. Herranen, T. Markkanen, S. Nurmi, and A. Rajantie, Phys. Rev. Lett. 115, 241301 (2015).

[32] J. Kearney, H. Yoo, and K. M. Zurek, Phys. Rev. D 91, 123537 (2015).

[33] L. A. Anchordoqui, V. Barger, H. Goldberg, X. Huang, D. Marfatia, L. H. M. da Silva, and T. J. Weiler, Phys. Rev. D 92, 063504 (2015).

[34] F. Kahlhoefer and J. McDonald, J. Cosmol. Astropart. Phys. 11 (2015) 015.

[35] Y. Ema, K. Mukaida, and K. Nakayama, J. Cosmol. Astropart. Phys. 10 (2016) 043.

[36] Y. Ema, K. Mukaida, and K. Nakayama, Phys. Lett. B 761, 419 (2016).

[37] N. Okada and D. Raut, Eur. Phys. J. C 77, 247 (2017).

[38] K. Urbanowski, Theor. Math. Phys. 190, 458 (2017).

[39] A. Stachowski, M. Szydowski, and K. Urbanowski, Eur. Phys. J. C 77, 357 (2017).

[40] L. N. Mihaila, J. Salomon, and M. Steinhauser, Phys. Rev. Lett. 108, 151602 (2012).

[41] K. G. Chetyrkin and M. F. Zoller, J. High Energy Phys. 06 (2012) 033.

[42] F. Bezrukov, M. Y. Kalmykov, B. A. Kniehl, and M. Shaposhnikov, J. High Energy Phys. 10 (2012) 140.

[43] J. Elias-Miro, J. R. Espinosa, G. F. Giudice, G. Isidori, A. Riotto, and A. Strumia, Phys. Lett. B 709, 222 (2012).

[44] D. Buttazzo, G. Degrassi, P. P. Giardino, G. F. Giudice, F. Sala, A. Salvio, and A. Strumia, J. High Energy Phys. 12 (2013) 089.

[45] V. Branchina and E. Messina, Phys. Rev. Lett. 111, 241801 (2013).

[46] V. Branchina, E. Messina, and A. Platania, J. High Energy Phys. 09 (2014) 182.
[47] V. Branchina, E. Messina, and M. Sher, Phys. Rev. D 91, 013003 (2015).

[48] V. Branchina and E. Messina, Europhys. Lett. 117, 61002 (2017).

[49] N. Haba, H. Ishida, R. Takahashi, and Y. Yamaguchi, Nucl. Phys. B900, 244 (2015).

[50] P. M. Ferreira and B. Swiezewska, J. High Energy Phys. 04 (2016) 99.

[51] N. Chakrabarty and B. Mukhopadhyaya, Eur. Phys. J. C 77, 153 (2017).

[52] E. Bentivegna, V. Branchina, F. Contino, and D. Zappalá, J. High Energy Phys. 12 (2017) 100.

[53] G. Isidori, V. S. Rychkov, A. Strumia, and N. Tetradis, Phys. Rev. D 77, 025034 (2008).

[54] V. Branchina, E. Messina, and D. Zappalá, Europhys. Lett. 116, 21001 (2016).

[55] A. Rajantie and S. Stopyra, Phys. Rev. D 95, 025008 (2017).

[56] J. R. Espinosa, J.-F. Fortin, and M. Trépanier, Phys. Rev. D 93, 124067 (2016).

[57] M. Kamionkowski and J. March-Russell, Phys. Lett. B 282, 137 (1992).

[58] A. G. Dias, A. C. B. Machado, C. C. Nishi, A. Ringwald, and P. Vaudrevange, J. High Energy Phys. 06 (2014) 037.

[59] L. Di Luzio, E. Nardi, and L. Ubaldi, Phys. Rev. Lett. 119, 011801 (2017).

[60] H. P. Nilles, Phys. Rep. 110, 1 (1984).

[61] A. Andreassen, W. Frost, and M. D. Schwartz, Phys. Rev. Lett. 113, 241801 (2014).

[62] L. Di Luzio and L. Mihaila, J. High Energy Phys. 06 (2014) 079 .

[63] A. Andreassen, W. Frost, and M. D. Schwartz, Phys. Rev. D 91, 016009 (2015).

[64] M. Endo, T. Moroi, M. M. Nojiri, and Y. Shoji, J. High Energy Phys. 11 (2017) 074.

[65] A. Andreassen, W. Frost, and M. D. Schwartz, Phys. Rev. D 97, 056006 (2018).

[66] S. Chigusa, T. Moroi, and Y. Shoji, Phys. Rev. Lett. 119, 211801 (2017).

[67] P. B. Arnold and S. Vokos, Phys. Rev. D 44, 3620 (1991).

[68] S. R. Coleman and E. J. Weinberg, Phys. Rev. D 7, 1888 (1973).

[69] O. Antipin, M. Gillioz, J. Krog, E. Mlgaard, and F. Sannino, J. High Energy Phys. 08 (2013) 034.

[70] P. Burda, R. Gregory, and I. Moss, J. High Energy Phys. 06 (2016) 025.

[71] C. Vafa, arXiv:hep-th/0509212.

[72] N. Arkani-Hamed, L. Motl, A. Nicolis, and C. Vafa, J. High Energy Phys. 06 (2007) 060.

[73] G. Obied, H. Ooguri, L. Spodyneiko, and C. Vafa, arXiv: 1806.08362.

[74] T. W. Grimm, E. Palti, and I. Valenzuela, J. High Energy Phys. 08 (2018) 143.

[75] M. Carena, J. R. Ellis, A. Pilaftsis, and C. E. M. Wagner, Nucl. Phys. B586, 92 (2000).

[76] A. Djouadi, Phys. Rep. 459, 1 (2008).

[77] N. Arkani-Hamed and S. Dimopoulos, J. High Energy Phys. 06 (2005) 073.

[78] G. F. Giudice and A. Romanino, Nucl. Phys. B699, 65 (2004); B706, 487 (2005). 
[79] B. Garbrecht, C. Pallis, and A. Pilaftsis, J. High Energy Phys. 12 (2006) 038.

[80] M. Dine, L. Randall, and S. D. Thomas, Nucl. Phys. B458, 291 (1996).

[81] L. E. Ibanez and G. G. Ross, Nucl. Phys. B368, 3 (1992).

[82] T. Gherghetta, C. F. Kolda, and S. P. Martin, Nucl. Phys. B468, 37 (1996).

[83] C. Panagiotakopoulos and A. Pilaftsis, Phys. Rev. D 63, 055003 (2001).

[84] L. M. Krauss and F. Wilczek, Phys. Rev. Lett. 62, 1221 (1989).
[85] T. Banks and M. Dine, Phys. Rev. D 45, 1424 (1992).

[86] R. Kallosh, A. D. Linde, D. A. Linde, and L. Susskind, Phys. Rev. D 52, 912 (1995).

[87] P. Byakti, D. Ghosh, and T. Sharma, J. High Energy Phys. 01 (2018) 015.

[88] E. Witten, Commun. Math. Phys. 100, 197 (1985).

[89] P. Horava and E. Witten, Nucl. Phys. B460, 506 (1996).

[90] I. Antoniadis, N. Arkani-Hamed, S. Dimopoulos, and G. R. Dvali, Phys. Lett. B 436, 257 (1998).

[91] L. Randall and R. Sundrum, Phys. Rev. Lett. 83, 3370 (1999). 